\title{
Density reduction and diffusion in driven two-dimensional colloidal systems through microchannels
}

\author{
P. Henseler, ${ }^{1, *}$ A. Erbe, ${ }^{1,2, \dagger}$ M. Köppl, ${ }^{1}$ P. Leiderer, ${ }^{1}$ and P. Nielaba ${ }^{1}$ \\ ${ }^{i}$ Universität Konstanz, Fachbereich für Physik, 78457 Konstanz, Germany \\ ${ }^{2}$ Universität Konstanz, Zukunftskolleg, 78457 Konstanz, Germany
}

\begin{abstract}
The behavior of particles driven through a narrow constriction is investigated in experiment and simulation. The system of particles adapts to the confining potentials and the interaction energies by a self-consistent arrangement of the particles. It results in the formation of layers throughout the channel and of a density gradient along the channel. The particles accommodate to the density gradient by reducing the number of layers one by one when it is energetically favorable. The position of the layer reduction zone fluctuates with time while the particles continuously pass this zone. The flow behavior of the particles is studied in detail. The velocities of the particles and their diffusion behavior reflect the influence of the self-organized order of the system.
\end{abstract}

\section{INTRODUCTION}

Pedestrians in a pedestrian zone [1], ants following a trail to food places and many other systems of interacting entities, which are moving in opposite directions to each other, show a prominent feature, namely the formation of lanes along the direction of their motion. This formation of lanes has been studied theoretically for colloidal particles in three dimensions [2-4] as well as in 2 dimensional systems [5-7]. These examples indicate that flow of particles can have a substantial influence on the structure formation of a system of interacting particles. Experimental studies on such systems have not been performed up to date, first hints of a lane formation transition could be seen in a three-dimensional system of oppositely charged colloids driven in opposite directions by application of an external electric field [8]. Studies of people in panic (for example trying to escape from a building) show the influence of constrictions on such moving ensembles.

A system of two dimensionally confined moving colloidal particles also resembles the classical analog of a quantum point contact in mesoscopic electronics $[9,10]$ or in metallic single atom contacts [11-13]. These contacts exhibit transport in electronic channels due to quantization effects. Such quantum channels can be seen as similar to the layers in the macroscopic transport, since both occur due to the interaction of the particles with the confining potential. A classical version of a similar scenario can be built on a liquid helium surface, which is loaded with charges. For such a system the formation of layers has been reported as well [14]. The change in the number of such layers in the vicinity of a constriction has been predicted from Langevin dynamics simulations of Yukawa particles [15].

In biological systems the transport of interacting particles through narrow constrictions is of high importance for many

\footnotetext{
*Present address: d-fine GmbH, Opernplatz 2, 60313 Frankfurt, Germany; peter.henseler@d-fine.de

'Present address: Forschungszentrum Dresden-Rossendorf, Institute for Ion Beam Physics and Materials Research, 01328 Dresden, Germany; a.erbe@fzd.de
}

processes, for example for the size selectivity of transport in ion channels [16]. The complexity of such systems allows only to make simplified statements on the underlying physics governing such phenomena. Experimentally easily accessible model systems can reveal many of the underlying processes. In the context of microfluidics and "lab-on-a-chip" devices one is interested in nonequilibrium transport and mixing phenomena on the microscopic scale [17].

In this paper we present a two-dimensional system of moving, superparamagnetic particles. The interaction energies between the particles and therefore the effective temperature of the system can be set by application of an external magnetic field. The phase behavior of these particles in two dimensions has been studied extensively without external fields [18-21] and under the influence of external fields [22-29]. In addition to this, it has been shown that confinement of these particles in a narrow channel leads to the formation of layers, in order to conform to the boundaries set by the hard walls $[30,31]$. The effect of the type of confinement on the ordering of a crystal confined to stripes of finite width was analyzed using Monte Carlo simulations by Ricci and co-workers [32-34]. The number and the stability of these layers change as the density, the interparticle interactions, or the boundary conditions at the wall are varied. In this work we address the question how these layers change when the particles are subject to a driven motion along the channel. In order to investigate this moving state, we first study the properties of a static system using Brownian dynamics simulations. Based on these results, the moving system is characterized and the results are compared to an experimental system of superparamagnetic particles moving through a lithographically defined channel.

\section{EXPERIMENTAL SETUP}

The particles are constricted to a narrow channel connecting two reservoirs, which are defined on a substrate using UV-lithography [35]. Images produced with a scanning electron microscope (SEM) of such a channel setup are shown in Fig. 1. 


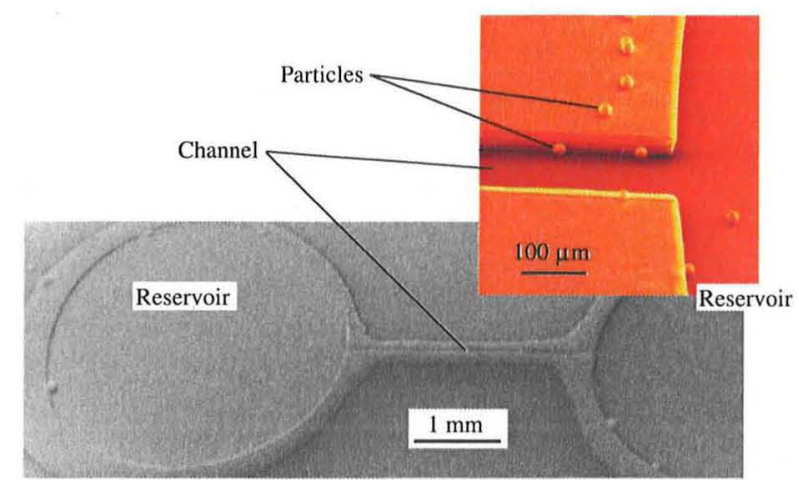

FIG. 1. (Color online) SEM images of the full channel geometry connecting two reservoirs and an enlargement of the channel entrance/exit region. Also some dried colloidal particles can be seen. During measurement the particles outside of the channel are removed so that they don't influence the particle transport within the channel [36].

Channel geometries of various width and length have been produced. The simulation results are compared to a channel being $60 \mu \mathrm{m}$ wide, $2.7 \mathrm{~mm}$ long and having channel walls of about $5 \mu \mathrm{m}$ in height. The channel is filled with a suspension of superparamagnetic particles of diameter $4.5 \mu \mathrm{m}$ in water (Dynabeads). Identical particles have been used previously and characterized in [23]. A summary of the properties of these colloidal particles is given in Table I.

Gravity confines the particles to the bottom surface of the channel due to the density mismatch between the colloids and the surrounding water. An external uniform magnetic field $\mathbf{B}=B \hat{\mathbf{z}}$ is applied perpendicular to the bottom surface. As a consequence the colloids form a monolayer in the $x-y$ plane with induced parallel dipole moments in $z$ direction giving rise to a purely repulsive pair-wise particle interaction. The strength of the repulsive force at distance $r_{i j}=\mid \mathbf{r}_{i}$ $-\mathbf{r}_{j} \mid$ is given by

$$
V_{i j}\left(r_{i j}\right)=\left(\mu_{0} / 4 \pi\right) M^{2} / r_{i j}^{3},
$$

with the magnetic dipole moments $M=\chi_{\mathrm{eff}} B$ of the particles. The importance of the pair interaction can be characterized by the dimensionless interaction strength

$$
\Gamma=\mu_{0} M^{2}(\pi n)^{3 / 2} /\left(4 \pi k_{B} T\right),
$$

where $n$ denotes the (overall) particle number density, $k_{B}$ the Boltzmann constant, $\mu_{0}=1.257 \times 10^{-6} \mathrm{Vs} / \mathrm{Am}$ is the magnetic permeability of free space and $T$ the temperature. For an unbounded equilibrated two-dimensional (2D) system

TABLE I. Particle properties of the Dynabeads used in the experiment.

\begin{tabular}{lcc}
\hline \hline Diameter & $\sigma$ & $4.55 \pm 0.1 \mu \mathrm{m}$ \\
Mass density & $\rho_{\text {colloid }}$ & $1.6 \mathrm{~g} / \mathrm{cm}^{3}$ \\
Particle mass & $m$ & $(7.6 \pm 0.1) \times 10^{-14} \mathrm{~kg}$ \\
Saturation magnetization & $M_{0}$ & $(5.7 \pm 0.4) \times 10^{-13} \mathrm{Am}^{2}$ \\
Effective susceptibility & $\chi_{\text {eff }}$ & $7.5 \times 10^{-11} \mathrm{Am}^{2} / \mathrm{T}$ \\
\hline \hline
\end{tabular}

which forms a triangular lattice, the particle number density can be written in terms of the lattice constant $\tilde{a}$ as

$$
n=\frac{2}{\sqrt{3}} \frac{1}{\tilde{a}^{2}} .
$$

So, $\Gamma=\left(\frac{2 \pi}{\sqrt{3}}\right)^{3 / 2} V_{i j}(\widetilde{a}) /\left(k_{B} T\right)$ is the mean dipolar interaction energy of Eq. (1) in terms of the thermal energy. Accordingly, the applied magnetic field $B$ which is connected to the magnetization via $M=\chi_{\text {eff }} B$ plays the role of an effective inverse temperature.

The external magnetic field is the dominant magnetic field in this system as it is obvious from the large particle separations in the video microscopy snapshot of Fig. 10(a) and the mutual induction between the colloids is negligible. Thermal and magnetically induced fluctuations of the positions of the particles perpendicular to the plane of inclination are less than $10 \%$ of the particle diameter and can be neglected. Tilting of the whole channel setup induces transport of the colloids from one reservoir into the other due to gravity. An alternative driving method would be the application of an in-plane magnetic-field gradient.

Before starting experiments the system is set up exactly horizontal. The particles are allowed to sediment to the bottom surface and arrange in the equilibrium configuration within several hours. Before tilting the whole apparatus the particles are either all confined in one reservoir (by use of laser tweezers) or uniformly distributed along the channel and within both reservoirs. In the experiment an inclination of $\alpha_{\text {exp }}=0.6 \mathrm{deg}$ is chosen, where the system is in a gravitationally driven nonequilibrium situation, but not yet in the regime of plug flow. This inclination results in an average particle drift velocity $v_{\text {drift }} \approx 0.035 \mu \mathrm{m} / \mathrm{s}$. A typical snapshot from the experiment of the particles moving along the channel is given in Fig. 10(a).

The particle trajectories are tracked with a video microscope. The repetition time of the video microscope setup is $10 \mathrm{~s}$. All experiments are made at room temperature $T \approx 295 \mathrm{~K}$.

In the experiment the number density of the colloids is defined as the number of colloids divided by the area of the 2D channel within the field of view of the video microscope accessible to the centers of the colloids. This dimensionless parameter $\Gamma$ was introduced by Zahn $e$ t al. [18], who studied experimentally the so-called Kosterlitz-Thouless-HalperinNelson-Young (KTHNY) phase transition in an unbounded two-dimensional equilibrium system of superparamagnetic particles, to characterize the system state. They found that for $\Gamma<\Gamma_{i} \equiv 52.9$ the system behaves such as a fluid, and for $\Gamma>\Gamma_{m} \equiv 60.9$ the system forms a triangular lattice. For the $\Gamma$ values in between they observed the so-called KTHNY or hexatic phase.

In the experiments described below a magnetic field of strength $B=0.24 \mathrm{mT}$ is applied, corresponding to $\Gamma \approx 72$ which is in the solid state region of the phase diagram.

\section{SIMULATION DETAILS}

We conduct Brownian dynamics (BD) simulations of a two-dimensional microchannel setup in order to investigate 
the flow behavior of the colloidal particles within the channel, reflecting the experimental situation, and by varying systematically various parameter values of inclination, over-all particle density, and channel width. The equation of motion for an individual colloidal particle is given by an overdamped Langevin equation. This approach neglects hydrodynamic interactions as well as the short-time momentum relaxation of the particles. Both approximations are fully justified in the current experimental context. Typical momentum relaxation times are on the order of $100 \mu \mathrm{s}$ and therefore much shorter than the repetition time of the video microscopy setup (10 s) used in the experiment. Thus the colloidal trajectories $\mathbf{r}_{i}(t)=\left[x_{i}(t), y_{i}(t)\right](i=1, \ldots, N)$ are approximated by the stochastic position Langevin equations with the Stokes friction constant $\xi$

$$
\xi \frac{d \mathbf{r}_{i}(t)}{d t}=-\nabla_{\mathbf{r}_{i}} \sum_{i \neq j} V_{i j}\left(r_{i j}\right)+\mathbf{F}_{i}^{\mathrm{ext}}+\tilde{\mathbf{F}}_{i}(t) .
$$

The right-hand side includes the sum of all forces acting on each particle, namely, the particle interaction, the constant driving force along the channel $\mathbf{F}_{i}^{\text {ext }}=m g \sin (\alpha) \hat{\mathbf{x}}$ and the random forces $\widetilde{\mathbf{F}}_{i}(t)$. The latter describe the collisions of the solvent molecules with the $i$ th colloidal particle and in the simulation are given by a Wiener process, i.e., by random numbers with zero mean, $\left\langle\widetilde{\mathbf{F}}_{i}(t)\right\rangle=0$, and variance $\left\langle\widetilde{F}_{i \alpha}(t) \widetilde{F}_{i \beta}(0)\right\rangle=2 k_{B} T \xi \delta(t) \delta_{i j} \delta_{\alpha \beta}$. The subscripts $\alpha$ and $\beta$ denote the Cartesian components. The effective mass $m$ of the particles is determined by the density mismatch between the particles and the solvent. These position Langevin equations are integrated forward in time in a Brownian dynamics simulation using a finite time step $\Delta t$ and the technique of Ermak $[37,38]$.

Particles are confined to the channel by hard walls in $y$ direction and at $x=0$ (channel entrance). These walls are realized both as ideal elastic hard walls and as proposed in [39], where a particle crossing the wall is moved back along the line perpendicular to the wall until contact. Both realizations result in the same flow behavior. Also we performed simulations with the particles at the wall kept fixed. The channel end is realized as an open boundary. To keep the over-all number density in the channel fixed, every time a particle leaves the end of the channel a particle is inserted at a random position (avoiding particle overlaps) within the first $10 \%$ of the channel, acting as a reservoir. A cutoff of $10 \sigma$ was used along with a Verlet next neighbor list [38]. Checks of particle overlaps are included in the simulation, but for all ordered systems we never found two overlapping particles.

Starting from a random particle distribution within the channel, we first calculate an equilibrium configuration $\left(\mathbf{F}_{i}^{\mathrm{ext}}=0\right)$ of a closed channel with ideal hard walls. Afterwards we apply to the configuration of uniform density the external driving force and allow the system to reorganize for $10^{6}$ time steps, before we evaluate the configurations. The time step $\Delta t=7.5 \times 10^{-5} \tau_{B}$ is used, with $\tau_{B}=\xi \sigma^{2} / k_{B} T$ being the time necessary for a single, free particle in equilibrium to diffuse its own diameter $\sigma$. We choose $\xi=3 \pi \eta \sigma$, with $\eta$ denoting the shear viscosity of the water. The simulations are done with 2000-4500 particles, for a channel geometry of $L_{x}=800 \sigma$ and $L_{y}=(9-12) \sigma$, and $\chi_{\text {eff,sim }}=3 \times 10^{-11} \mathrm{Am}^{2} / \mathrm{T}$. Thus external magnetic fields $B=0.1-1.0 \mathrm{mT}$ and a total particle density of $n=0.4 \sigma^{-2}$ correspond to $\Gamma \approx 21.34-2134$.

\section{EQUILIBRIUM PROPERTIES OF THE CHANNELS}

Equilibrated configurations of systems confined to a microchannel are used as starting configurations for our analysis of the transport behavior. This guarantees that at the beginning of the transport simulation the particles are uniformly distributed over the whole channel. First, we compare some results found for the $2 \mathrm{D}$ microchannels in equilibrium (the external driving force is switched off) with the results of Haghgooie and co-workers [30,31,40].

During the equilibration process the channel beginning at $x=0$ and the channel end at $x=L_{x}$ are either closed by ideal hard walls, or periodic boundary conditions are applied in $x$ direction. By doing so, we assure that no transport is initiated due to the boundary conditions used. The simulation start parameters are chosen in such a way that they closely reflect the situation of the experiment. In all simulations the area $L_{x} \cdot L_{y}$ is defined as the region accessible to the particle centers. This is the reason, why in the following simulation snapshots the $y$ positions of the edge particle centers coincide with the channel boundary. When comparing the channel widths in the simulation to the widths of the channel in the experiment, one has to add the particle diameter $\sigma$ resulting in $L_{y}^{\text {exp }}=L_{y}+\sigma$, e.g., a channel with $L_{y}=10 \sigma$ corresponds to a channel of $L_{y}^{\exp }=11 \sigma=49.5 \mu \mathrm{m}$ for the particles used. The equilibration process is usually started from a uniform random particle distribution over the whole channel. But to avoid a physical instability of the starting configuration the particle separations are limited to values greater than $0.7 \sigma$. For very dense systems this initialization method of course breaks down and we start from a hexagonally ordered configuration.

\section{A. Influence of the confinement}

The triangular lattice is the high-density equilibrium configuration of an unbounded 2D system. Here, we analyze how the confinement modifies the resulting equilibrium configurations. We submitted simulation runs to determine the equilibrium configuration in dependence of the channel width $L_{y}$ for a superparamagnetic system with $B=0.5 \mathrm{mT}$ applied and the global particle density $n=0.4 \sigma^{-2}$ which corresponds to $\Gamma=533.74$ and is deep in the solid phase region. Typical snapshots of representative parts of the equilibrium configurations being obtained are shown in Fig. 2. Shown are the regions $300 \sigma \leq x<600 \sigma$ of a channel with a total length of $L_{x}=800 \sigma$. Notice, that the channel widths are stretched by a factor of about 6.67 .

Obviously, whether an ordered or a perturbed configuration is formed strongly depends on the channel width $L_{y}$. For certain channel widths it is energetically favorable for the system to arrange into what we call layers. Right of configuration snapshots of Fig. 2 the equilibrium density profiles perpendicular to the channel walls are plotted. They are cal- 


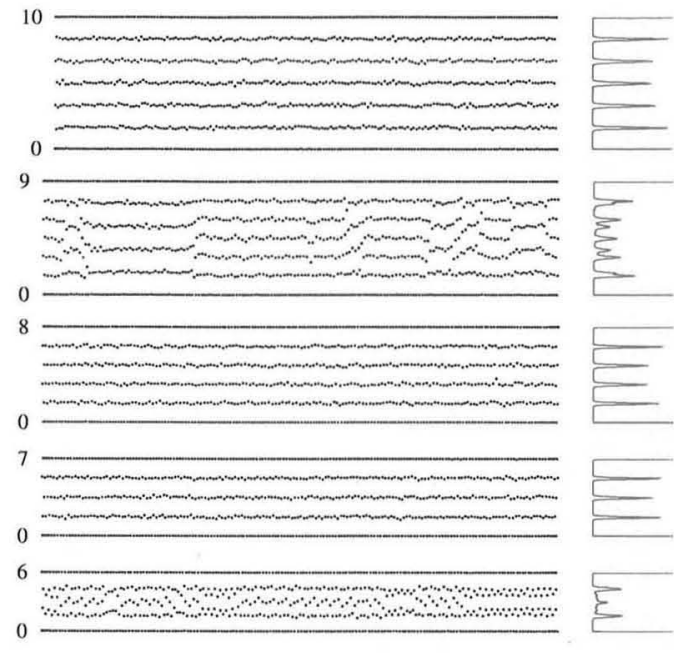

FIG. 2. (Color online) Typical simulation snapshots of partitions with length $300 \sigma$ of equilibrated configurations for a dipolar system $(B=0.5 \mathrm{mT}, \Gamma=533.74)$ and a selection of channel widths $(10 \sigma$ $9 \sigma, 8 \sigma, 7 \sigma$, and $6 \sigma$ from top to bottom). The channel widths are stretched by a factor of about 6.67. All configurations have the overall particle density $n=0.4 \sigma^{-2}$. The curves at the right of each configuration snapshot show averaged density profiles across the channel. For clarity reason, the large magnitude peaks at the walls have been truncated at a fixed peak height.

culated by taking the average over 2000 equilibrium configurations. For the channel widths $L_{y}=7 \sigma, 8 \sigma$ and $10 \sigma$ the peaks of these density histograms are well separated and occur at almost regular spacing across the channel. These properties are the signature of a well-defined layered structure parallel to the walls. For the channel widths $L_{y}=6 \sigma$ and $L_{y}$ $=9 \sigma$ the system cannot equilibrate into such a single layered structure over the full channel and only partial layering is visible in the configuration snapshots. Such a confinement induced layering phenomenon is in agreement with the results for liquid-dusty plasmas [41] and the results of the simulations of Haghgooie [30].

The channel widths of $10 \sigma, 9 \sigma, 8 \sigma, 7 \sigma$, and $6 \sigma$ correspond to the widths $6.80 R, 6.12 R, 5.44 R, 4.76 R$, and $4.08 R$ in units of $R=1.471 \sigma$, which is the expected separation of layers for the unbounded system. A pronounced boundary induced layer structures was also seen for much wider channels, for example for the case $L_{y}=20 \sigma$.

Even for wide channels of width $L_{y}=20 \sigma$ a clearly boundary induced layered structure occurs for a system at $\Gamma$ $=133.44$.

\section{B. Layer order parameter}

The number of layers forming within the channel can be identified by an appropriate local order parameter. We therefore divide the channel of width $L_{y}$ into several bins in $x$ direction each containing $n_{\text {bin }}$ particles and define for different number of layers $n_{l}$ the so-called layer order parameter

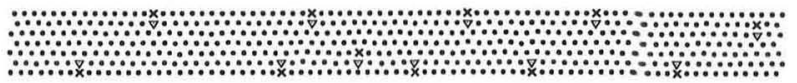

FIG. 3. Simulation: Snapshot of a partition $(450 \sigma \leq x \leq 580 \sigma)$ of the equilibrium defect configuration for the system with $L_{y}$ $=10 \sigma$ as shown in Fig. 2. Full circles (-) mark the bulk particles with six nearest neighbors and particle on the wall with four nearest neighbors, symbol $\times$ corresponds to fivefold symmetry (or threefold if on the wall), and symbol $\nabla$ to sevenfold symmetry (or fivefold if on the wall).

$$
\Psi_{\text {layer }, n_{l}}=\left|\frac{1}{n_{\text {bin }}} \sum_{j=1}^{n_{\text {bin }}} e^{\mathrm{i}\left(2 \pi\left(n_{l}-1\right) / L_{y}\right) y_{j}}\right|,
$$

which is unity for particles distributed equidistantly in $n_{l}$ layers across the channel width starting at $y=0$, and significantly smaller for the nonlayering case.

In Fig. 3 all defects within a partition of the equilibrated configuration are marked. For the layered system state the defects always occur in pairs (forming a dislocation) and are located predominantly close to the walls with quite a regular spacing. Due to the purely repulsive nature of the particle pair interaction the edge particles are pressed against the confining ideal hard walls as it is obvious from the high peaks of very small width at the boundary of Fig. 2. These defects along the walls are a consequence of a (slightly) higher line density of the edge particles compared to the bulk layers [30]. For example, for the system with $L_{y}=10 \sigma$ of Fig. 3 the line density of the wall layers is about $6 \%$ higher than of the nearest bulk layers. Edge layers have only a single neighbor layer whereas bulk layers have two. Putting an additional particle into a layer results both in stronger interaction within this layer and of this layer with its neighboring layers. Thus, it is energetically favorable for the system to have defects along the wall instead within the bulk, because there the involved energy barrier is lower.

The appearance of dislocations along the wall was also seen in $[24,42]$, where we systematically analyzed the equilibrium configurations constricted within a circular hard-wall confinement for dipolar and screened Coulomb pair interaction as function of the particle number. In these systems the particles arrange in multiple circles and the defects occur due to the bending of the lattice in presence of the curved boundary. This is in contrast to the situation here, where the planar walls give no need for the lattice to bend.

So, we can conclude that the layer order parameter is more suitable than $\psi_{6}(x)$ for the detection of layered structures and changes therein, because it is insensitive to defects close to the wall.

\section{Effects of channel width and interaction strength}

Two independent simulation parameters have a strong influence on the state of the dipolar system laterally confined between two parallel ideal hard walls. These are the wall separation $L_{y}$ and the dimensionless interaction strength $\Gamma$. In the following, we will compare these dependencies for our simulation parameters qualitatively with the results of Haghgooie [40]. 


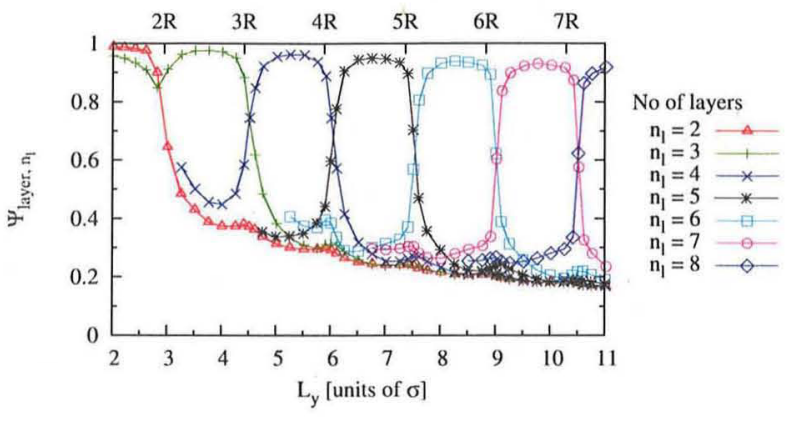

FIG. 4. (Color online) Simulation: the layer order parameter as function of the channel width. The simulation parameters are: $B$ $=0.25 \mathrm{mT}, \Gamma=133.44, R=1.471 \sigma, L_{x}=800 \sigma, n=0.4 \sigma^{-2}$, and periodic boundaries in $x$ direction.

\section{System state dependency on the channel width}

The influence of the channel width on the system state is analyzed by examining the behavior of the global layer order

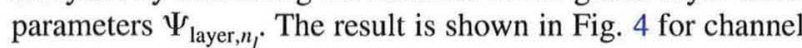
widths between $2 \sigma$ and $11 \sigma$. The global layer order parameters as function of the channel widths show for different number of layers $n_{l}$ distinct response regimes where their values are close to one. On top of the graph, we also indicated the channel width in units of the length scale $R$. Clearly, the change in the number of layers happens with a period of $\sim R$. For integer multiples of $R$ the system is not in a layered configuration, but in the transition between two layered structures. This means that the confinement induced optimal layer separation is smaller than the separation $R$ expected for the unbounded system, in agreement with the findings of equilibrium studies by Haghgooie et al. [40].

The above scenario can be confirmed by looking at the bulk defect concentration

$$
C_{\text {defect }}^{b} \equiv \frac{N_{\text {defect }}^{b}}{N^{b}}
$$

which is defined as the ratio of the number $N_{\text {defect }}^{b}$ of bulk particles with either more or less than six nearest neighbors and the total number $N^{b}$ of bulk particles. All particles with a distance greater then $0.5 \sigma$ are defined as bulk particles. In Fig. $5 C_{\mathrm{defect}}^{b}$ is plotted as function of the channel width for identical simulation parameters as used above. The concentration of defects in the bulk shows an oscillatory behavior with a period of $\sim R$. The peak positions indicate the channel widths where the system cannot equilibrate into a layered structure, and the positions of the minima coincide with stable layer configurations. This behavior is in good agreement with the results of Haghgooie as can be seen from taking slices of constant $\Gamma_{H}$ in Fig. 6 of [40]. The decay in the maxima values for increasing channel width indicates the approach to the bulk behavior.

\section{Time evolution of the defect configuration}

In Fig. 6 the time evolution of the defect concentration $C_{\text {defect }}^{b}$ of the bulk particles during an equilibration run is explicitly plotted for a selection of $\Gamma$ values for a channel of

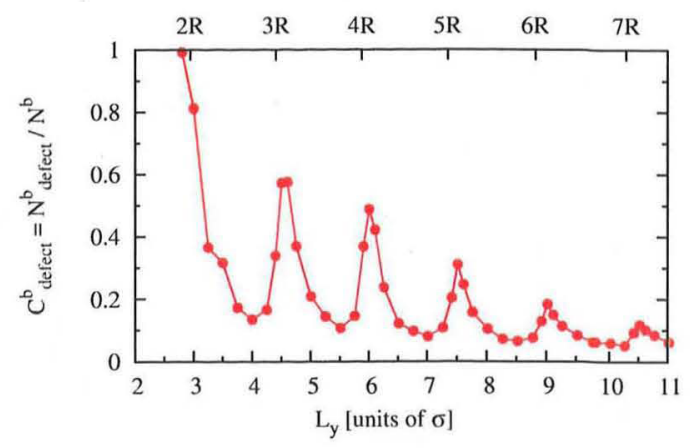

FIG. 5. (Color online) Simulation: the bulk defect concentration as function of the channel width for identical simulation parameters as in Fig. 4. Please note that for $L_{y} \leq 2 R$, the channel contains only two layers at the walls, thus $C_{\mathrm{defect}}^{b}=1$.

width $L_{y}=10 \sigma$. All runs are started from a random particle distribution. After a time of $10 \tau_{B}$ the defect concentration remains unchanged for all $\Gamma$ values. For $45.0<\Gamma<80$, i.e., for the transition region between the liquid and the solid state, the equilibration process is slower than for the other values. The fluctuations increase near the phase boundary. These effects are consistent with the results of Haghgooie [40] obtained for an unbounded system.

\section{System state dependency on the interaction strength}

In Fig. 7 we show density profiles perpendicular to the confining walls for the two channel widths $L_{y}=9 \sigma$ and $L_{y}$ $=10 \sigma$ at four values of $\Gamma$. On the left-hand side both systems are liquid whereas on the right-hand side they are both in the solid state. These density histograms are obtained by taking the average over 3500 configurations in equilibrium. The system characteristics are very different depending on the $\Gamma$ value and the channel width $L_{y}$. For high $\Gamma$ values, where the system is in the solid state, the density profile for the channel width $L_{y}=10 \sigma$ is sharply peaked at the positions of the seven layers. On decrease in the interaction strength $\Gamma$ these peaks broaden and have a Gaussian profile down to a value of $\Gamma$ $\approx 65$. The central peaks show greater broadening than the peaks at the wall, i.e., the system melts first in the center of

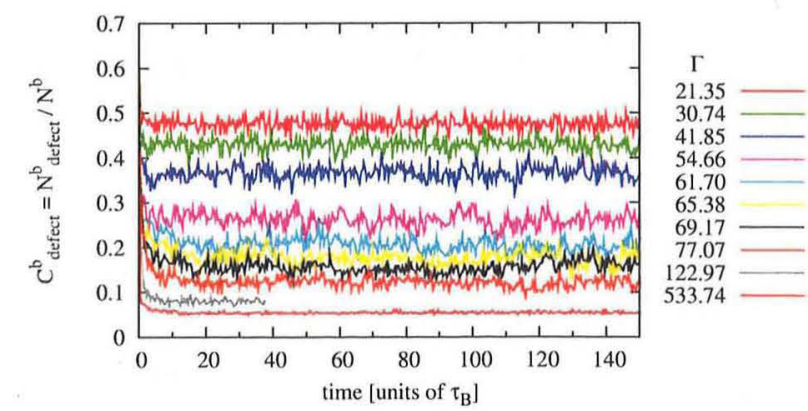

FIG. 6. (Color online) Simulation: time evolution of the defect concentration $C_{\text {defect }}^{b}$ for a channel with $n=0.4 \sigma^{-2}, N=4000$, and $L_{y}=10 \sigma$ kept fixed. The particle interaction strength is modified via the applied magnetic field $B$ having values between 0.1 and $0.5 \mathrm{mT}$. The $\Gamma$ values increase from the top to the bottom curve. 


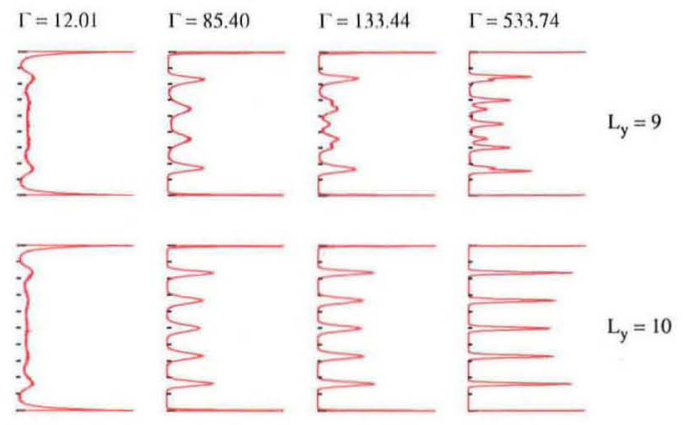

FIG. 7. (Color online) Simulation: density profiles perpendicular to the walls for $L_{y}=9 \sigma$ and $L_{y}=10 \sigma$ in dependence of $\Gamma$. Again, the peaks at the walls are truncated for better clarity.

the channel. Even for low $\Gamma$ values as $\Gamma \approx 12.01$, where the unbounded system would be deep in the liquid state, the particles at the wall are still relatively localized in their $y$ positions. A clear density minimum between the colloids in the edge layer and the colloids of the central region can always be identified. For the channel width $L_{y}=9 \sigma$ the melting scenario is different. The peak profile is less pronounced for $\Gamma=533.74$ and there is less order across the channel. A mixture between a structure of six and of seven layers is indicated by the positions of the peak maxima. The structure of seven layers is favored more, because the peaks connected to a structure of seven layers are more pronounced than the remaining peaks related to six layers. Decreasing $\Gamma$ again leads to a broadening of the peaks and the structure with six layers becomes more favorable $(\Gamma=133.44)$. The unbounded system would be well in the solid state at this value at this interaction strength. For $\Gamma=85.40$ only the peaks related to six layers remain, and for $\Gamma=12.01$ no significant qualitative difference to the situation for the channel of width $10 \sigma$ exists.

These changes in the peak characteristics of the density profile across the channel of width $L_{y}=9 \sigma$ is also reflected in the behavior of the layer order parameters in Fig. 8 for $n_{l}$ $=6$ and $n_{l}=7$ layers on variation in the interaction strength. $\Psi_{\text {layer }, n_{l}=6}$ exhibits a maximum at about $\Gamma=90$, and strongly decreases for higher $\Gamma$ values whereas the values of $\Psi_{\text {layer }, n_{l}=7}$ increase to values of about 0.8 .

Piacente and co-workers [43] studied the structural, dynamical properties, and melting of a quasi-one-dimensional system of charged particles, interacting through a screened

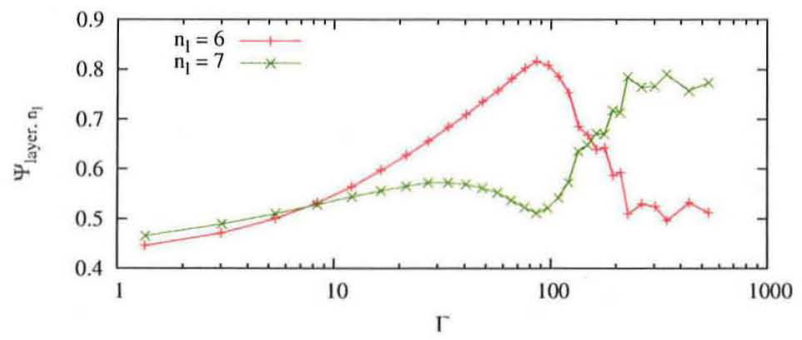

FIG. 8. (Color online) Simulation: comparison of the dependency on the interaction strength $\Gamma$ of the global layer order parameter $\Psi_{\text {layer }, n_{l}}$ with $n_{l}=6$ and $n_{l}=7$ for the channel width $L_{y}=9 \sigma$.

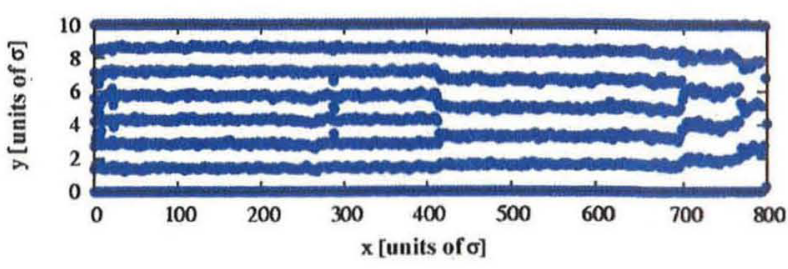

FIG. 9. (Color online) Simulation: full channel snapshot for a channel with ideal hard walls $(\Gamma=533.74, \alpha=0.2 \mathrm{deg})$ after $10^{6}$ BD simulation steps having reached a stationary nonequilibrium state. Note, that the scaling on the $y$ axis is stretched by a factor of 20 compared to the $x$ axis scaling.

Coulomb potential in equilibrium. This system is related to our situation, but a different particle interaction potential is used and the particles are confined in $y$ direction by a parabolic potential. They also find a rich structural phase diagram with different layered structures as function of the screening length $\kappa_{D}^{-1}$ and the electron density $n_{e}$ of the system.

\section{TRANSPORT BEHAVIOR OF COLLOIDS IN MICROCHANNELS}

Now, we want to address the transport behavior of colloids confined to such microchannels as described in the previous section. The colloids are driven by the application of an external driving force $\mathbf{F}$ and thus form a system in nonequilibrium. This driving can be of gravitational origin as in our case, or due to the presence of an electrical or magnetic field or an osmotic pressure difference between both channel ends. To match the experimental situation closely, we will concentrate mainly on colloids with repulsive dipolar pair interaction driven by gravity. First we introduce the effect of dynamical rearrangement of the colloids during their flow along the channel. We call this effect layer reduction.

\section{A. Layer reduction}

A first impression of the particle arrangement under the influence of an external driving field give the Figs. 9 and 10 which depict typical configuration snapshots from simulation and experiment. The particles move along the channel from left to right in the positive $x$ direction.

The external magnetic field strength $B$, which is responsible for the strength of the pair interaction and the overall particle number density $n$ are chosen in such a way, that the confined equilibrium system is hexagonally ordered. This is true also for the unbounded system under identical conditions. Figure 9 is a representative snapshot taken in the simulation of the full channel having the length $L_{x}=800 \sigma$ $=3.6 \mathrm{~mm}$. The first $10 \%$ of the channel act as reservoir. In the experiment the channel length is $L_{x}=444.4 \sigma=2.0 \mathrm{~mm}$. The strength of the constant driving force $\mathbf{F}^{\mathrm{ext}}=F \mathbf{e}_{x}$ can either be specified directly or by definition of the inclination $\alpha$ resulting in $F=m g \sin \alpha$. Under the influence of external driving the particles still form layers. Additionally we observe, both in experiment and in simulation, a decrease in the number of layers in the direction of motion $[36,44]$. The layer transitions are clearly visible in Fig. 9, where they are 


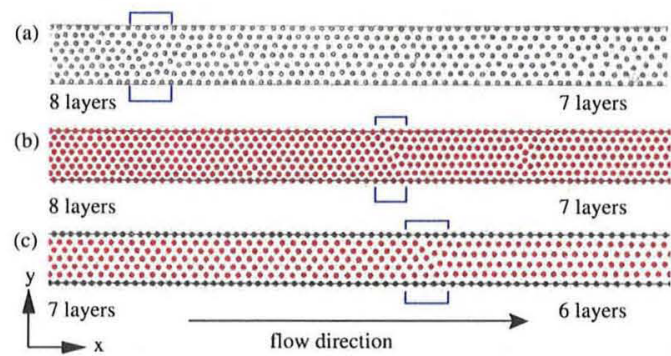

FIG. 10. (Color online) (a) Experiment: video microscopy snapshot of colloidal particles moving along the lithographically defined channel. The channel partition shown has the size $(692 \times 60) \mu \mathrm{m}$ $=(153.8 \times 13.33) \sigma$, and the interaction strength is $\Gamma \approx 72$, and $\alpha$ $=0.6 \mathrm{deg}$. (b) Simulation: snapshots for a channel with ideal hard walls $[(573.3 \times 45) \mu \mathrm{m}=(127.4 \times 10) \sigma, \Gamma=640.5]$, (c) the same as in (b) with the particles at the walls (marked green) kept fixed $[(573.3 \times 45) \mu \mathrm{m}, \Gamma=5026, \alpha=0.2 \mathrm{deg}]$. The rectangles mark the region of the layer reduction zone.

located at $x \approx 420 \sigma$ from eight to seven layers, at $x \approx 700 \sigma$ from seven to six layers, and at $x \approx 770 \sigma$ from six to five layers.

The images of Fig. 10 show in enlargement the part of the channel near the region of layer reduction zone being marked by the rectangle. The video microscope snapshot of Fig. 10(a) is taken from the experiment [36]. The small white spots at the particle centers allow for precise tracking of the particle trajectories with the video microscope. Similar snapshots we get from our BD simulations with either comoving [Fig. 10(b)] or fixed edge particles [Fig. 10(c)]. In these two subfigures the filled circles represent the particles at their real size relative to the channel width. For these highly ordered systems the layer transitions take place on the scale of only a few particle diameters.

\section{B. Density gradient along the channel}

The simulation snapshots above are taken after a time long enough for the system to reach a stationary nonequilibrium situation. Applying the external driving force to the equilibrated channel configuration leads to the build up of a particle density gradient along the channel. This is an effect of the chosen boundary conditions at the channel entrance and exit, which leads to a pressure difference between both channel ends. After about $10^{6} \mathrm{BD}$ time steps this density gradient does not change any more, which is the signature of a stationary state. The exact origin of the density gradient is given by details of the particle-particle interactions in combination with the driving force and will be subject of a separate publication.

To study the robustness of the formation of the density gradient and its connection to the layer reduction in our system of gravitationally driven particles, we performed simulations for a variety of inclinations $\alpha=0.0 \mathrm{deg}-10.0 \mathrm{deg}$ keeping the overall particle density fixed at $n=0.4 \sigma^{-2}$. The resulting stationary nonequilibrium density profiles along the channel are shown in Fig. 11. They are calculated from histograms of the $x$ positions of 1000 configurations in station-

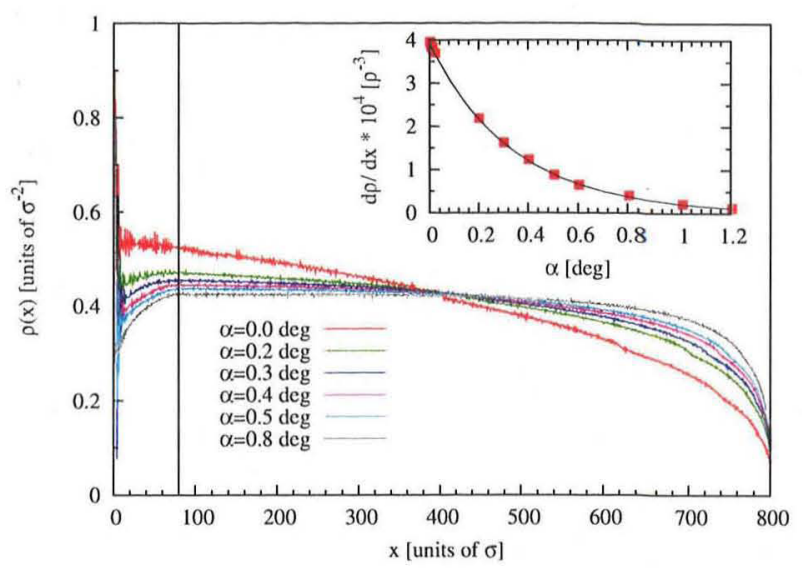

FIG. 11. (Color online) Simulation: stationary non equilibrium density histograms along the channel for several values of the slope $\alpha\left(\Gamma=533.75\right.$ and $\left.n=0.4 \sigma^{-2}\right)$. The vertical line at $x=80 \sigma$ marks the right end of the reservoir, i.e., the maximum $x$ value up to where particles are inserted randomly. At $x=80 \sigma$ the angle $\alpha$ increases from the top to the bottom curve. The inset shows the density gradient in the interval $x \in[150,600] \sigma$ as a function of the inclination $\alpha$ being obtained from linear fits to the density histograms. Here the line connecting the data points serves as a guideline to the eye.

ary nonequilibrium. A very significant decrease in the local density occurs for $x>700 \sigma$, which is caused by the open boundary at $x=800 \sigma$. The region $0 \leq x<80 \sigma$ acts as reservoir, where new particles are inserted at random position whenever a particle drops out at the end of the channel. To avoid unnecessary high perturbations due to random particle reinsertion in the reservoir the channel is closed at $x=0 \sigma$ by a semipermeable ideal hard wall.

All density profiles show a nearly linear density gradient in the interval $x \in[150,600] \sigma$, which is maximal for $\alpha$ $=0 \mathrm{deg}$ (cf. inset of Fig. 11). Even at $\alpha=0 \mathrm{deg}$ a (osmotic) pressure difference between both channel ends exists for the boundary conditions used, and a small particle flux is induced. For inclinations $\alpha>1.0 \mathrm{deg}$ the density gradient becomes almost zero. For these inclinations the driving force dominates, and we find plug flow of the particles without layer reduction. A decrease in the inclination (driving force) gives rise to an increase in the density gradient. Under nonplug flow condition we find a self-induced arrangement of the particles to a nearly hexagonal lattice and the occurrence of layer reductions with the particles moving across.

\section{Dynamical Properties}

\section{Drift velocity}

It is also interesting to study the average overall drift velocity as function of the driving force. The result is shown in Fig. 12.

For $\alpha>0.5 \mathrm{deg}$ the particle flow is dominated by the driving force. This is the regime of plug flow, where the particles move with 


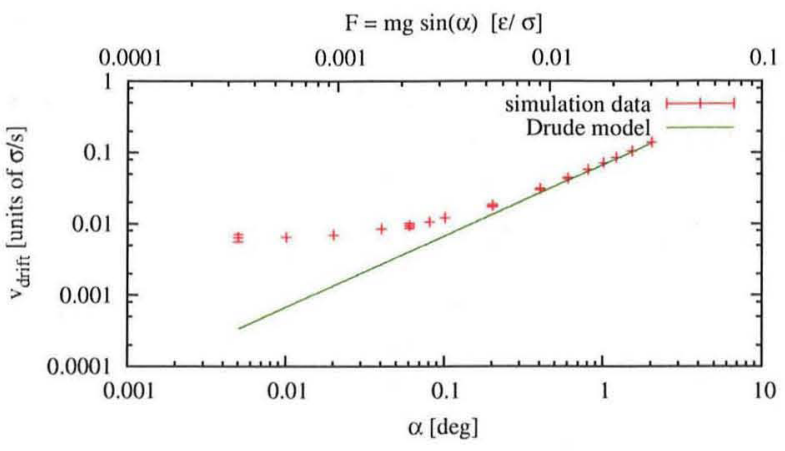

FIG. 12. (Color online) Simulation: average particle drift velocity in the interval $x \in[200,770] \sigma$ as function of the inclination $\alpha$ or equivalently the driving force $\mathbf{F}=m g \sin (\alpha) \mathbf{e}_{x^{*}}\left(L_{x}=800 \sigma, L_{y}=10 \sigma\right.$, $n=0.4 \sigma^{-2}$, and $\left.\Gamma=533.74\right)$. The solid line is the expected drift velocity for noninteracting particles due to the external driving (Drude model).

$$
\left\langle v_{\text {drift }}\right\rangle_{\text {Drude }}=\frac{m g}{\xi} \sin \alpha,
$$

as expected for noninteracting particles. Such a dependency was formulated by P. Drude [45] for electrical conduction to explain the transport of electrons in metals. For $\alpha$ $<0.5$ deg the average drift velocity deviates from the expectation of the Drude model. Interestingly, for these inclinations the particles move faster than expected. The Drude model is based on a friction dependent mobility coefficient, only. For inclinations $\alpha<0.2 \mathrm{deg}$ the diffusion behavior of the particles has to be taken into account, too. Therefore, the interplay of the small drift and of the diffusion behavior gives rise to a change in the mobility in $x$ direction.

Particles moving along the channel get accelerated. This becomes obvious from Fig. 14, where histograms of the drift velocity together with Gaussian fits in different $x$-regions of the channel are plotted. All hydrodynamic interactions are neglected. Generally, the particle velocities $v_{x}$ in $x$ direction are normally distributed about the average drift velocity. For the angle $\alpha=0.2 \mathrm{deg}$ the average drift velocity is $\left\langle v_{\text {drift }}\right\rangle$ $\approx 0.081 \mu \mathrm{m} / \mathrm{s}=0.018 \mu / \mathrm{s}$. In the experiment an inclination of $\alpha_{\exp }=0.6 \mathrm{deg}$ was chosen, which results in $\left\langle v_{\text {drift }}\right\rangle$ $\approx 0.035 \mu \mathrm{m} / \mathrm{s}=0.0078 \mathrm{\sigma} / \mathrm{s}$. The velocities of the particles in the experiment are lower as compared to simulations, possibly due to the influence of hydrodynamic interactions. The comparison between edge particles and bulk particles shows the effect of layer changes of the particles on the velocities. As mentioned in Sec. IV dislocations are present along the walls. These dislocations lead to an increased number of layer changes for the edge particles. During the layer transition the particles move in $y$ direction rather than $x$ direction; therefore, we expect to see a superposition of two velocity distributions in $x$ direction, one centered around zero for particles changing layers and one centered around the velocity of the particles in the edge layer. Figure 13 shows Gaussian fits for the bulk particles and the edge particles. It is apparent that the velocity distribution of the edge particles can be fit by a superposition of 2 Gaussian fits, resembling the par-

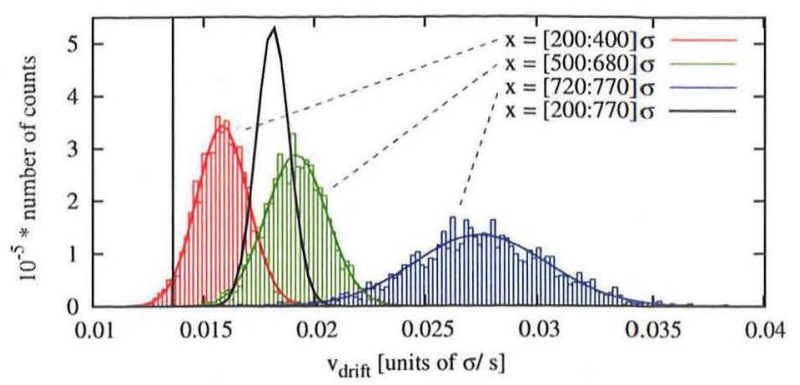

FIG. 13. (Color online) Simulation: drift velocity histograms of the particles in different channel regions. The vertical line marks the expected drift velocity for noninteracting particles according to the Drude model [cf. Eq. (7)].

ticles changing lanes (around $0 \mu \mathrm{m} / \mathrm{s}$ ) and moving straight $(0.031 \mu \mathrm{m} / \mathrm{s})$. The different velocities of bulk and edge layers are caused by the difference in density of the layers.

\section{Example particle trajectories}

The particles flow across the layer reduction zone (cf. Fig. 15), whereas the position of the layer reduction zone almost remains unchanged. We show in Fig. 15(a) representative particle trajectories for a selection of particles. These are marked in the configuration snapshots [Fig. 15(b)] at their beginning and the final location of the trajectories. The trajectories clearly show that we do not observe plug flow of a crystal, but rather a dynamic behavior of particles moving in layers and adapting to the external potential. The particles move a distance of about $60 \sigma$ whereas the layer transition stays located within $x \in[390,400] \sigma$.

The edge particles are pushed against the ideal hard walls at $y=0 \sigma$ and $y=L_{y}=10 \sigma$ by the repulsion of the inner particles of the channel. This is the reason for their minimal fluctuations perpendicular to the flow direction. The corresponding fluctuations of the nonedge layers are significantly larger, and a small increase in the mobility in $y$ direction with increasing wall separation is found. In the central region some particles change very abruptly from one layer to another whereas others shift more smoothly. The particles in

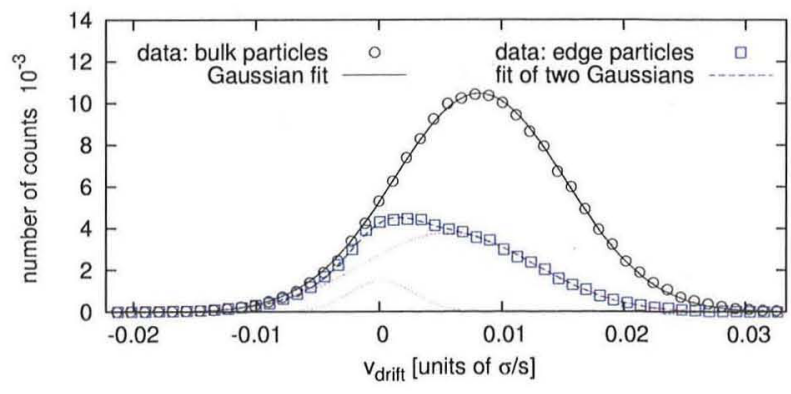

FIG. 14. (Color online) Experiment: drift velocity histograms of the particles in the full field of view. Shown are the histograms for the bulk and the edge particles, respectively. The data points of the bulk particles can be fitted well by a single Gaussian, whereas the edge particles need to be fitted by a superposition of two Gaussian functions. The results of the fits are indicated by the lines. 
(a)

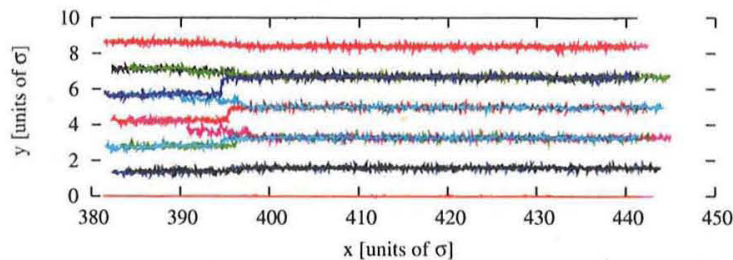

(b)

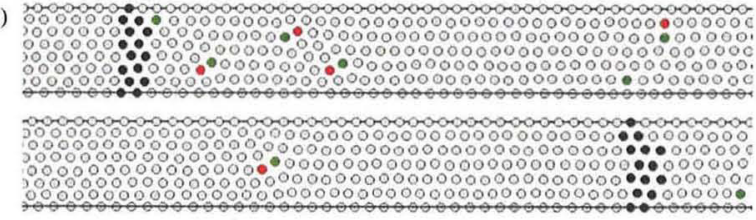

(c)

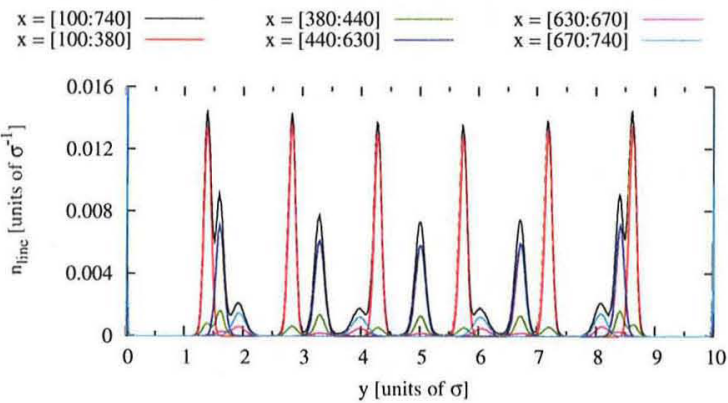

FIG. 15. (Color online) Simulation: (a) Example particle trajectories which show the dynamical rearrangement of the particles crossing the layer reduction zone from eight to seven layers. Shown are the trajectories for the time interval $\Delta t=37.5 \tau_{B}\left(\equiv 5 \times 10^{5} \mathrm{BD}\right.$ steps). (b) Corresponding snapshots of the starting and final configuration. The general (color) coding of the particles is described in the text. Additionally, all the particles which trajectories are shown in (a) have been marked (cluster of black particles). (c) Histograms of the $y$ positions within different $x$-regions evaluated for 1.5 $\times 10^{6} \mathrm{BD}$ steps. The peaks of the edge particles are truncated for clarity reason. The system parameters are identical to those of Fig. 9

the layers next to the edge layers only show a small and smooth change in their $y$ position. In the regions with fixed number of layers no particle transitions between layers are observed for our simulation parameters.

All particles are identical. In Fig. 15(b) the particles are (color) coded according to the number of nearest-neighbor particles they have. Bulk particles with six nearest neighbors and all edge particles are marked light gray, whereas particles in dark gray (red) have a fivefold symmetry and particles in medium gray (green) have a sevenfold symmetry of nearest neighbors. The actual number of nearest neighbors is determined using a Delaunay triangulation. In the start configuration three defect pairs (dislocations) are in the region of the layer transition form eight to seven layers, whereas in the final configuration the position of the layer reduction zone is connected to a single dislocation. The slightly higher density of the edge particles gives rise to the scattered medium gray (green) particles in the next edge layer.

For the same system we analyze the density profiles perpendicular to the walls within several subregions along the channel. Therefore we evaluate $1.5 \times 10^{6} \mathrm{BD}$ steps corre-
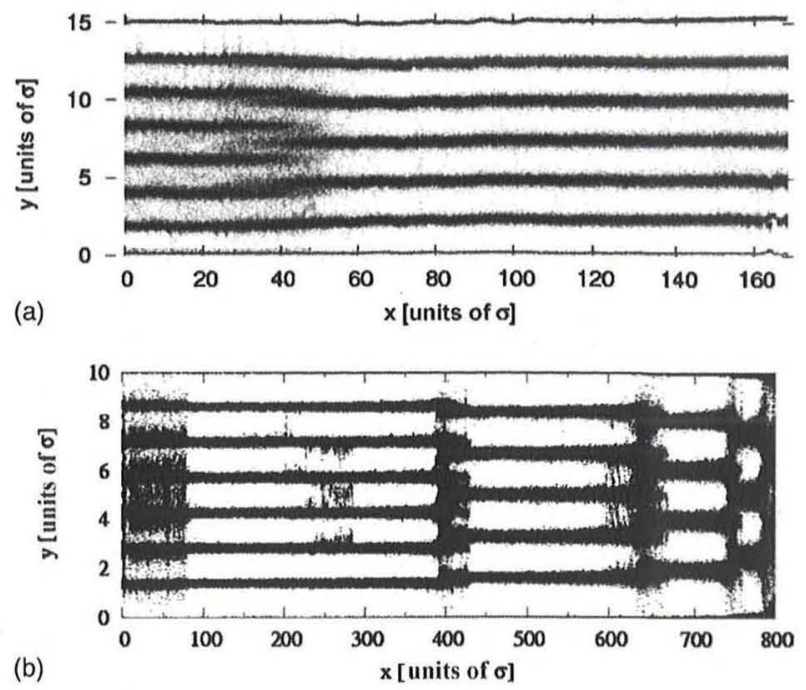

FIG. 16. (Color online) Superimposed configuration snapshots: (a) of the experiment, (b) of the simulation for $1.5 \times 10^{6} \mathrm{BD}$ steps, which corresponds to $\Delta t \approx 122.5 \tau_{B}$. $\left(B=0.5 \mathrm{mT}, \Gamma=533.74, L_{x}\right.$ $=800 \sigma, L_{y}=10 \sigma, n=0.4 \sigma^{-2}, \alpha=0.04 \mathrm{deg}$ )

sponding to a time interval of $\Delta t \approx 122.5 \tau_{B}$. The full density profile for $x \in[100,740] \sigma$ (black curve) is a superposition of several profiles connected to distinct layering. Highly ordered layer structures with sharply peaked density profiles occur for eight layers in $x \in[100,380] \sigma$ (red curve), seven layers in $x \in[440,630] \sigma$ (blue curve), and six layers in $x$ $\in[670,740] \sigma$ (cyan curve). The $x$ regions in between are the layer transition regions.

In Fig. 16 we explicitly plot the superposition of 1911 video microscopy snapshots of the experimental system and 3000 configuration snapshots used for the density profile evaluation above. In the experiment the particles move on average $\langle\Delta x\rangle \approx 670 \sigma$. The layer reduction zone is confined in the interval $x \in(5,50) \sigma$. In the simulation the particles have moved forward on average the distance $\langle\Delta x\rangle \approx 202 \sigma$, i.e., more than a quarter of the channel length. The layer transition positions remain located within an interval of length $45 \sigma$. The particles are inserted at a random position in the region $x \in(0,80) \sigma$. Perturbations of the configuration due to the random particle insertion heal after a few BD steps. Therefore the configuration for $x>90 \sigma$ is not influenced by this particle reinsertion method.

\section{Defect removal}

Sometimes "defects" remain after the point of layer reduction, which vanish on further flow. Here we call a defect a pair of particles having five and seven neighbors, respectively, which disturb a given layer configuration. These can be identified from dips they form in the local layer order parameter defined in Eq. (5) of the current configuration. Generally, small density gradients along the channel give rise to a larger number of defects than higher density gradients. This already is a hint on the close connection of the occurrence of layer transitions to the local number density. A defect can be neutralized by a particle changing into the edge 

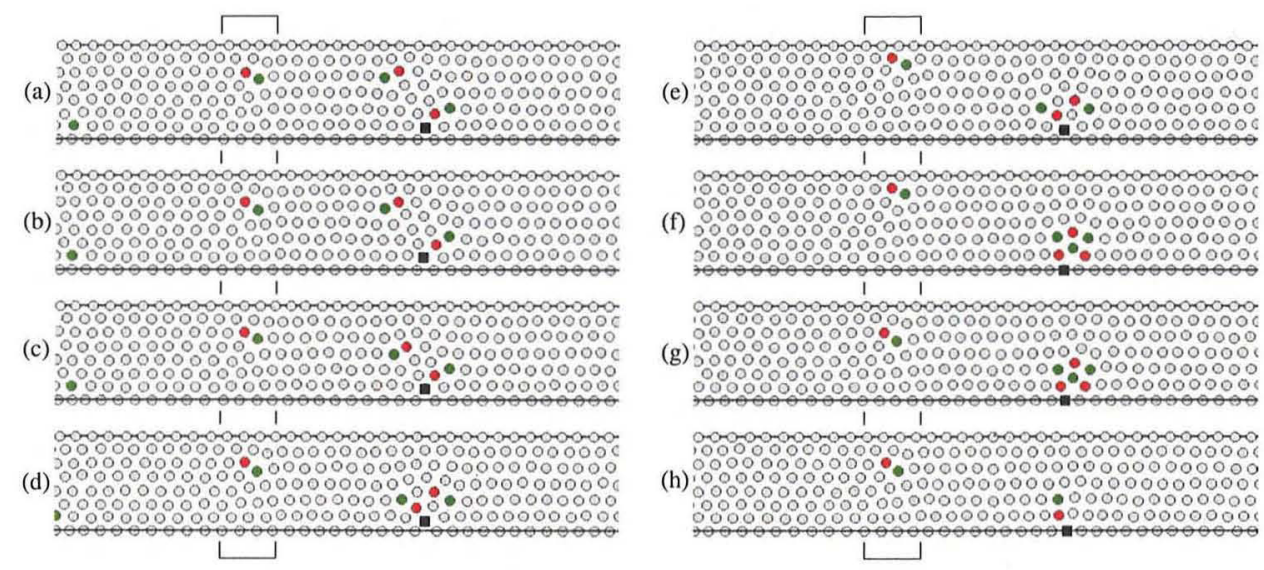

FIG. 17. (Color online) Simulation: the sequence of configuration snapshots (a) - (h) shows the process of a vanishing "defect" after the layer transition zone (marked by the black rectangle) due to the change of particle marked by the filled black square into the edge layer. The snapshots have been taken every $500 \mathrm{BD}$ time steps, i.e., $\Delta t=0.0375 \tau_{B}$.

layer. Such a neutralization process of two defects is shown in the sequence of configuration snapshots of Fig. 17 taken every $500 \mathrm{BD}$ time steps. The particle which is marked by a filled black square moves into the edge layer and thereby removes the perturbation of the layered structure of seven layers after the position of the layer transition region marked by the black rectangle. In the final snapshot $17(\mathrm{~h})$ seven unperturbed layers remain. Recognize that again the $x$ position of the layer reduction zone remains unchanged.

\section{Connection between the layer transition and the density gradient}

The reduction in the number of layers originates from a density gradient along the channel. The local particle density $\rho(x)$ inside the channel is shown in Fig. 18 together with the local lattice constants $d_{x}$ and $d_{y}$. The particle separations of

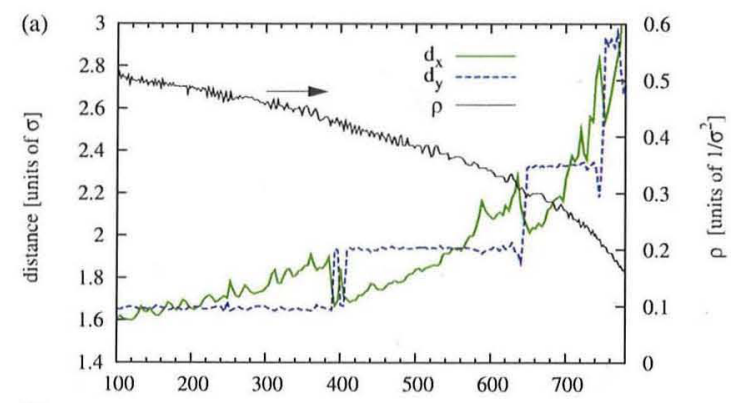

(b)

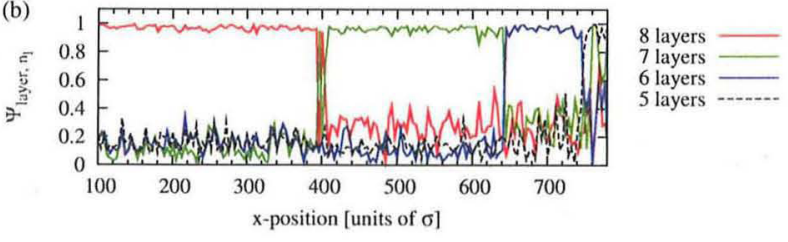

FIG. 18. (Color online) Simulation: (a) Local lattice constants $d_{x}$ and $d_{y}$ and local particle density $\rho$, (b) Corresponding local layer order parameters $\Psi_{\text {layer }, n}$. The system parameters are: $L_{x}=800 \sigma$, $L_{y}=10 \sigma, n=0.4 \sigma^{-2}, \alpha=0.2 \mathrm{deg}$, and $\Gamma=533.74$. neighboring particles in $x$ and $y$ direction are used to calculate the local lattice constant $d$ of the triangular lattice. Due to the density gradient along the channel, the ordered structure is not in its equilibrium configuration at all points along the channel. Thus the local lattice constant $d_{x}$, calculated from the particle separations in $x$ direction, can deviate from the local lattice constant $d_{y}$, calculated from the particle separations in $y$ direction and multiplication with the factor $2 / \sqrt{3}$. At the left end of the channel, $d_{x}$ increases to larger values than $d_{y}$, indicating that the ordered structure is stretched along the $x$ axis. At the position of the layer reduction the system changes back to a situation, where $d_{x}$ is smaller than $d_{y}$ by decreasing $d_{x}$ and increasing $d_{y}$ by about $20 \%$ simultaneously. These changes in separations compensate each other and result in a continuous change in the local density at the position of the layer reduction. The behavior of the system shows that the stretching of the ordered structure before the layer reduction causes an instability toward decreasing the number of layers. This decrease compresses the system along the $x$ direction, but apparently lowers the total energy of the system.

Layer transitions occur at almost identical values of the local particle density for various inclinations as can be seen in Fig. 19. Here the local lattice constant $d_{y}(x)$ is plotted as a function of the local particle density $\rho(x)$. Transitions from 8 to 7 layers occur when $\rho(x)$ becomes smaller than 0.42 , transitions from $7 \rightarrow 6$ layers for $\rho(x)<0.3$, and transitions from $6 \rightarrow 5$ layers for $\rho(x)<0.21$.

\section{Static stretching analysis}

The scenario above can be qualitatively confirmed by the following rough estimation: starting from an ideal triangular configuration with a given number of layers $\left(n_{l}\right)$ in a channel of fixed width, we calculate the potential energy per particle for different particle densities by scaling the channel length of the static configuration only. Plots of these energies per particle for different values of $n_{l}$ as function of the particle density $\rho$ are shown in Fig. 20. The intersection points, which are determined from linear approximation of both 


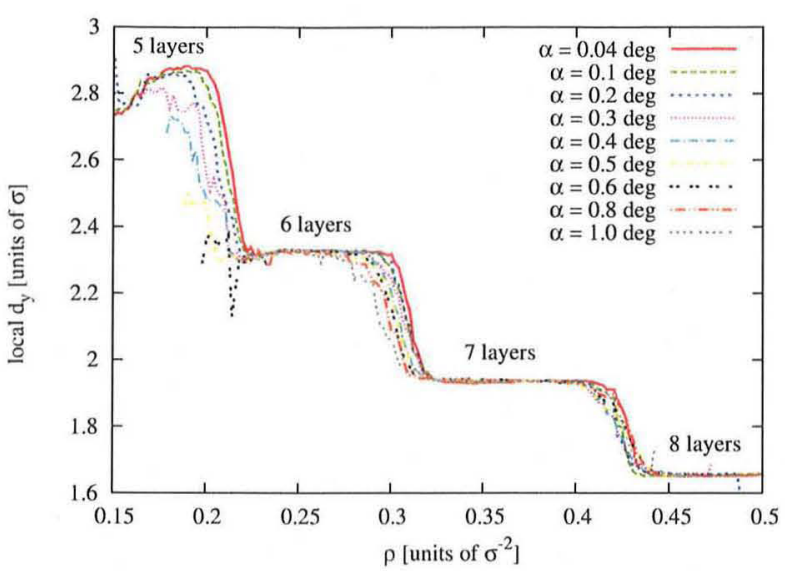

FIG. 19. (Color online) Simulation: local lattice constant $d_{y}$ as function of the local particle density $\rho$ for various inclinations $\alpha$.

curves in the region of intersection, serve as rough estimates of the densities at the layer transition points. For the given system the values for the transition $8 \rightarrow 7$ layers are: $\rho_{8 \rightarrow 7}$ $\approx 0.467 \sigma^{-2}$, and for the transition $7 \rightarrow 6$ layers: $\rho_{7 \rightarrow 6}$ $\approx 0.345 \sigma^{-2}$. So, we can conclude that a given layer structure is stable for up to slightly overstretched perfect triangular configurations [46].

They show clear intersection points, indicating that for a stretched configuration with $n_{l}$ layers in $x$ direction it can become energetically more favorable to switch to a compressed configuration with $\left(n_{l}-1\right)$ layers.

\section{Equilibrium configurations for confinement with nonparallel walls}

Also equilibrium BD simulations, i.e., simulations with no external driving force $\left(\widetilde{\mathbf{F}}_{i}^{\text {ext }}=0\right)$, of closed channels with nonparallel walls result in a density gradient in the direction of decreasing channel width. Here, confinement induced arrangement of the particles into different number of layers takes place. The particles just fluctuate about their equilib-

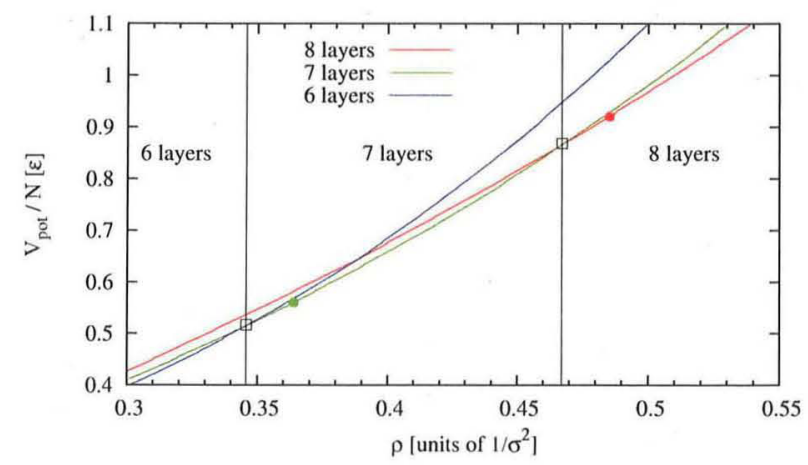

FIG. 20. (Color online) Result of the stretching analysis of static channel configurations of a channel with the width $L_{y}=10$ and dipolar pair interaction: shown are the potential energies per particle of different layer configurations as function of the particle density $\rho$. The full circles mark the perfect triangular lattices configurations of the respective number of layers.

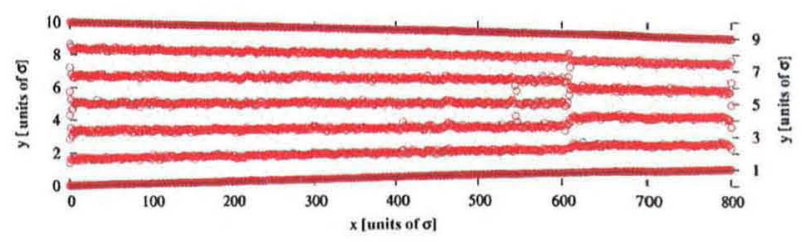

FIG. 21. (Color online) Simulation: Equilibrated configuration snapshot of a funnel geometry with opening angle $\alpha_{\text {Hopper }}$ $=0.143 \mathrm{deg}$. All walls are modeled as hard walls. No driving force is applied and the particles interaction is dipolar $(\Gamma=625.125)$.

rium positions. A snapshot of such an equilibrium configuration is shown in Fig. 21 where the confining funnel has the small opening angle $\alpha_{\text {Funnel }}=0.143 \mathrm{deg}$, i.e., over the full channel length of $L_{x}=800 \sigma$ the channel width decreases by $\Delta L_{y}=2 \sigma$. This kind of layer transition is a purely geometrical effect, whereas in the case of parallel walls and a constant longitudinal driving field the occurrence of the density gradient has a dynamical origin. In both cases the number of layers which form depends on the value of the local particle density $\rho(x)$.

\section{E. Comparison with the experiment}

The experimental result of the density gradient as well as the interparticle distances are shown in Fig. 22. The behavior closely resembles the behavior of the simulated system [cf. Fig. 18(a)]. The distance in $x$ direction, $d_{x}$, is continuously stretched while the distance in $y$ direction increases in a sharp step at the position of the layer reduction. The density decreases monotonously along the direction of motion of the particles by about $20 \%$.

In Fig. 23 snapshots of nonequilibrium defect configurations are shown both for the experiment (a) and for the simulation (b). They reveal that the system is nearly triangular left and right of the point of layer reduction. The change is marked by a single defect only. The number of layers is reduced one by one. Reductions by two or more layers have not been observed in experiment or in simulation. Naturally, this reduction produces a defect at the point of the transition. Since, the position of the layer reduction is mainly determined by the density gradient, its location remains stable

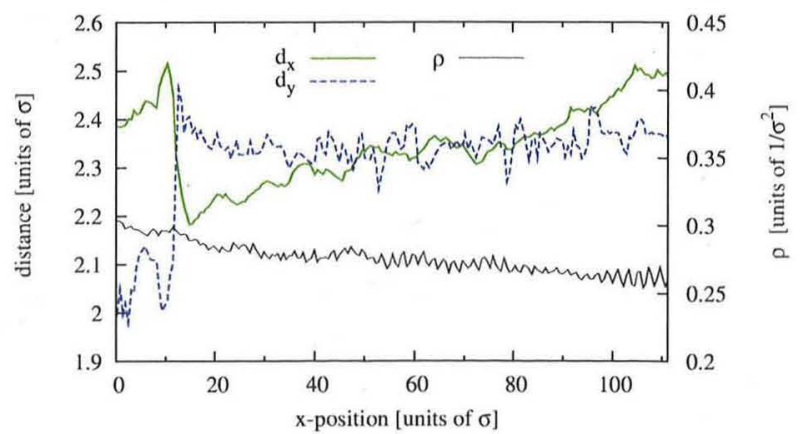

FIG. 22. (Color online) Experiment: local lattice constants $d_{x}$ and $d_{y}$ and local particle density. The results are obtained for the systems of which is shown in Fig. 10(a). 


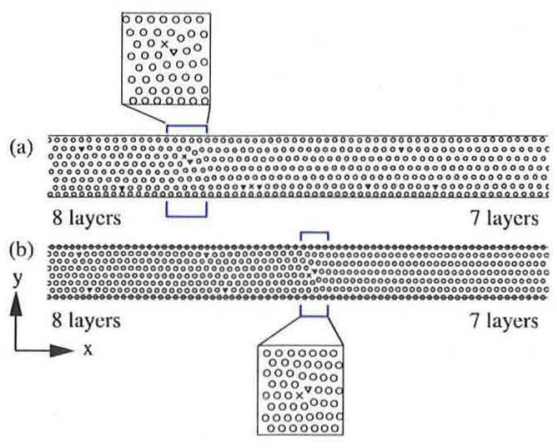

FIG. 23. (Color online) Snapshots of defect configurations obtained from a Delaunay triangulation of the particles moving in the channel. The particles are coded according to the number of their nearest neighbors. Open circles mark the bulk particles with six nearest neighbors and the edge particles, symbol $\times$ corresponds to a fivefold symmetry, and symbol $\nabla$ to a sevenfold symmetry. (a) Experiment: In order to minimize the effects of fluctuations on a short-time scale, 50 images have been averaged. (b) BD simulation for a channel with parallel walls.

with time on average. A more detailed analysis reveals, however, that the transition point oscillates back and forth around this average position. At the transition the driven particles in the bulk layers have to change the layer, causing the transition to move a little bit in direction of the flow. A particle changing into the edge layer can neutralize the defect of the transition locally. This causes a reconfiguration of the ordered structure, which in turn gives rise to repositioning of the layer reduction zone back to a region of higher density.

\section{F. Oscillatory behavior of the layer transition}

There are various ways of numerically localizing the position of the layer transition. One can either make use of the clear discontinuity of the local layer order parameters $\Psi_{\text {layer, }, n_{l}}(x, t)$ [cf. Eq. (5)] appropriate for the transition from $n_{l}$ to $n_{l}-1$ layers, or of the location of the discontinuity of the local lattice constant $d_{y}(x, t)$. The local orientational order parameter $\Psi_{6}$, which is often used for $2 \mathrm{D}$ systems [18], is not so significant for this system, as it is very sensitive to any perturbation of the sixfold symmetry. The first three methods have been used to study the position of the transition from eight to seven layers. The result is given in Fig. 24 .

In the nonequilibrium steady-state situation the position of the layer reduction zone oscillates about a certain $x$ position. This can also be seen in the experimental data, as shown in Fig. 25 (evaluated from the discontinuity in $d_{y}$ ).

Figures 24 and 25 show that the fluctuations are relatively small, indicating a situation close to a stationary state.

\section{G. Systems with screened Coulomb interaction}

In order to study the influence of the particle interaction range, we implemented the screened Coulomb (YHC) pair interaction potential
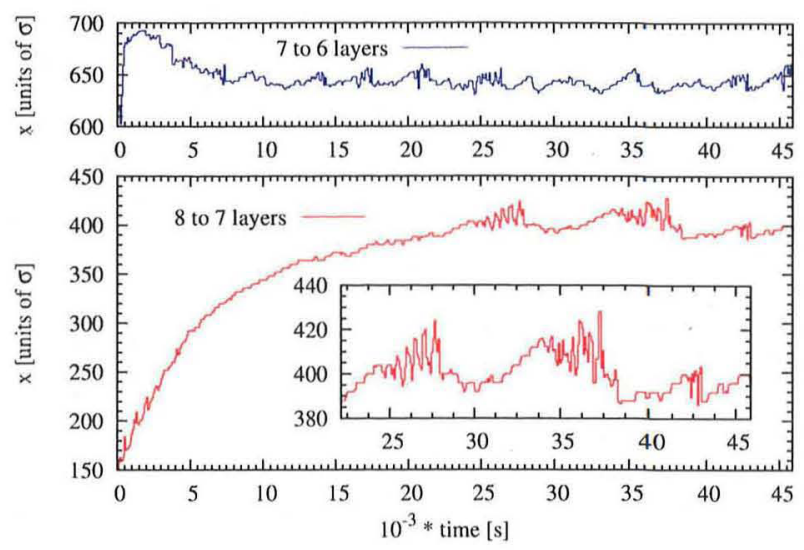

FIG. 24. (Color online) Simulation: movement of the $x$ position of layer transition, obtained from $\Psi_{\text {layer }, n}(x, t)$, for the transitions $8 \rightarrow 7$ layers and $7 \rightarrow 6$ layers. The system parameters are identical with those of Fig. 16.

$$
V_{i j}\left(r_{i j}\right)= \begin{cases}\infty: & r_{i j}<\sigma \\ V_{0} \frac{\exp \left[-\kappa_{D}\left(r_{i j}-\sigma\right)\right]}{r_{i j}}: & \sigma \leq r_{i j}<r_{\text {cut }}, \\ 0: & r_{i j} \geq r_{\text {cut }}\end{cases}
$$

with the inverse Debye screening length $\kappa_{D}$ which interpolates the potential between the hard core case (for $\kappa_{D} \rightarrow \infty$ ) and the unscreened Coulomb potential (for $\kappa_{D}=0$ ). $V_{0}$ is the value of the pair potential at contact which can be written as

$$
\beta V_{0}=\frac{Z^{2}}{\left(1+\kappa_{D} \sigma / 2\right)^{2}} \frac{\lambda_{B}}{\sigma},
$$

where $Z$ is the charge of the colloids and $\lambda_{B}$ $=e^{2} /\left(4 \pi \epsilon_{0} \epsilon_{s} k_{B} T\right)$ is the so-called Bjerrum length of the solvent with permittivity $\epsilon_{s}$.

Figure 26 is the analogous plot to Fig. 18(a) for a system of YHC particles with the contact value $\beta V_{0}=400$ and $\kappa_{D}$ $=4.0 \sigma^{-1}$. Under these simulation conditions no layer transition as for the dipolar system is found. For $x>450 \sigma$ the particles are ordered in seven layers, but for smaller values only a few islands of particles arranged in layers can be identified from the local order parameters along the channel in Fig. 26(b). The interaction range of a YHC system with $\kappa_{D}=4.0 \sigma^{-1}$ is much smaller than for the dipolar system, because of the stronger decay of the pair potential. This decay is also the reason for the large fluctuations of the local lattice

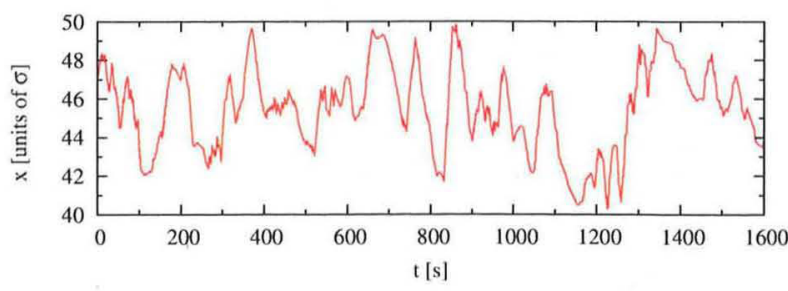

FIG. 25. (Color online) Experiment: movement of the $x$ position of layer transition for the transitions $8 \rightarrow 7$. 


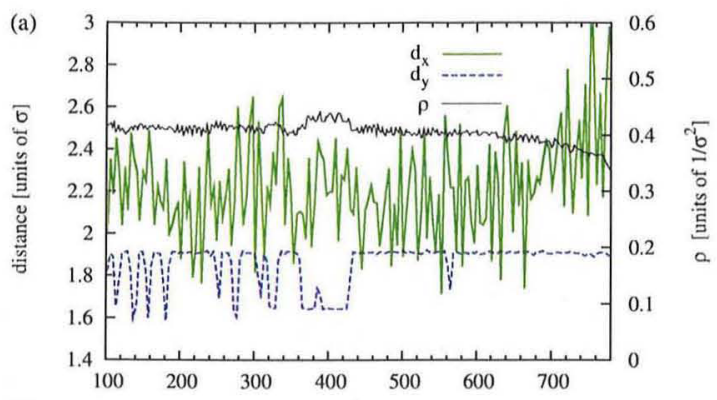

(b)

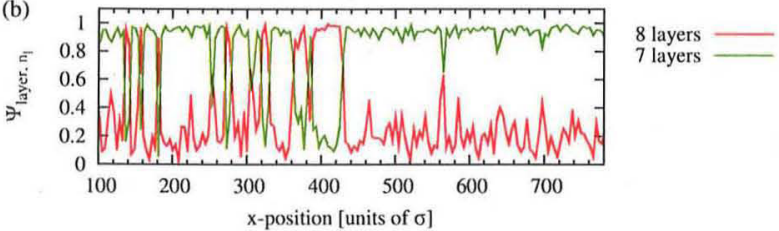

FIG. 26. (Color online) Simulation: (a) Local lattice constants $d x$ and $d_{y}$ and local particle density $\rho$ in the BD simulation of a system with screened Coulomb interaction. (b) Corresponding local layer order parameters $\Psi_{\text {layer }, n}$. The system parameters are: $L_{x}=800 \sigma$, $L_{y}=10 \sigma, n=0.4 \sigma^{-2}, \beta V_{0}=400, \kappa_{D}=4.0 \sigma^{-1}, \Gamma_{\mathrm{YHC}}=448.4$, and $\alpha$ $=0.2 \mathrm{deg}$.

constant $d_{x}$ in Fig. 26(a). A density gradient cannot form along the channel, and so no layer transition is found. The particles need to be strongly coupled with their neighboring particles to form a density gradient, i.e., the pair interaction range has to be at minimum of the order of the average particle spacing.

We studied the equilibrium density profiles perpendicular to the confining walls of a YHC system for a selection of $\kappa_{D}$ values and the value at particle contact $\beta V_{0}=50$. The total particle density is $n=0.45 \sigma^{-2}$ which corresponds to a packing fraction $\eta \approx 0.79$. For $\kappa_{D}=2 \sigma^{-1}$ boundary induced layering is found which becomes less pronounced for increasing $\kappa$, i.e., decreasing interaction range. For $\kappa_{D}>4 \sigma^{-1}$ the systems are fluid in the equilibrium state at this packing fraction, and only a depletion layer between the edge and the bulk particles was found.

The average particle separation of the unbounded system has the value $R \approx 1.38 \sigma$. For $\kappa_{D}>4 \sigma^{-1}$ the characteristic interaction range is $\sigma+\kappa_{D}^{-1}<1.25 \sigma$ which is smaller than $R$.

Now, we plot in Fig. 27 the superposition of 100 configurations with a time separation of $\Delta t=500 \mathrm{BD}$ steps after $1.4 \times 10^{6} \mathrm{BD}$ steps for the case of the alternative boundary condition in flow direction. The driving force corresponding to an inclination of $\alpha=0.1 \mathrm{deg}$ acts within $x \in[100,700] \sigma$. All four superimposed configurations show the formation of layers near the channel end at $x=700 \sigma$, where the particles enter the reservoir. In Fig. 27(a) the characteristic interaction range of the YHC pair potential is greater than the average particle spacing $R$. For this case we find multiple layer transitions from five layers up to eight layers along the channel. The system behavior is similar to the situation of the dipolar systems. With increasing values of $\kappa_{D}$ less layer transitions are observed. Figures 27 (b)-27(d) show increasing depletion zones at the channel start at $x=100 \sigma$. These depletion zones are followed by regions where the particles are in the liquid

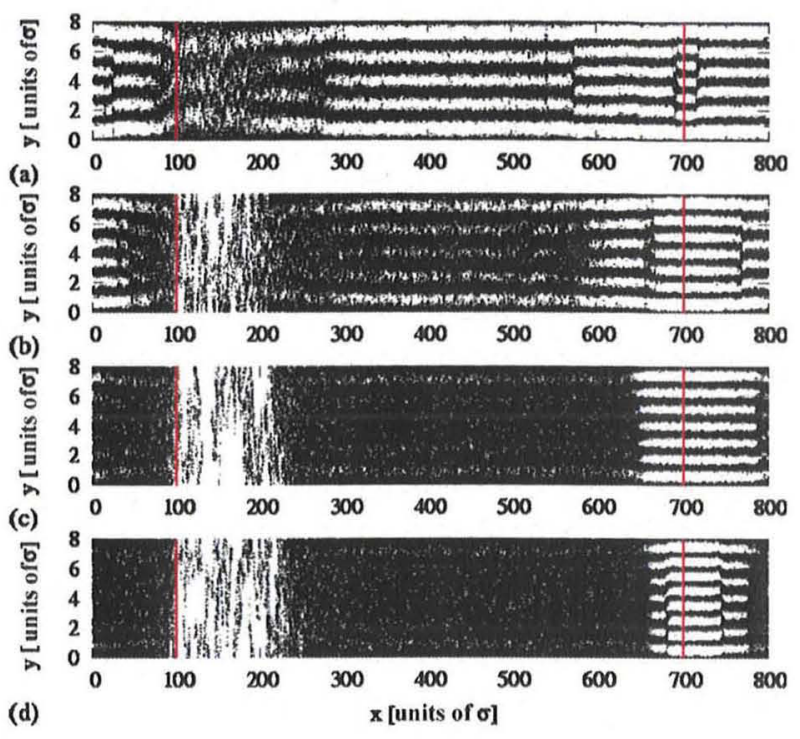

FIG. 27. (Color online) Simulation: superimposed configurations of systems with screened Coulomb pair interaction for a selection of inverse screening lengths: (a) $\kappa_{D}=2 \sigma^{-1}$, (b) $\kappa_{D}=4 \sigma^{-1}$, (c) $\kappa_{D}=8 \sigma^{-1}$, and (d) $\kappa_{D}=12 \sigma^{-1}$. The particle transport is induced for $x \in[100,700] \sigma$ by the inclination $\alpha=0.1 \mathrm{deg}$.

state. Notice, that for $\kappa_{D}=8 \sigma^{-1}$ and $\kappa_{D}=12 \sigma^{-1}$ the systems are in the liquid state in equilibrium, too (cf. Fig. 27). The corresponding density profiles in $x$ direction are given in Fig. 28.

The systems with $\kappa_{D}=8 \sigma^{-1}$ and $\kappa_{D}=12 \sigma^{-1}$ show a rapid increase in the local density from about $0.5 \sigma^{-2}$ up to values greater than $0.8 \sigma^{-2}$ in the interval $x \in[600,700] \sigma$. The particles under the influence of the constant driving force are blocked due to filling of the reservoir at the channel end. During the simulation run the particles pile up at the interface between the channel and the reservoir, because the particles of the channel are pushed into the reservoir but within the reservoir the particles diffuse almost freely due to the short range of the YHC interaction (high values of $\kappa_{D}$ ). This leads to a situation where the influx into the reservoir is

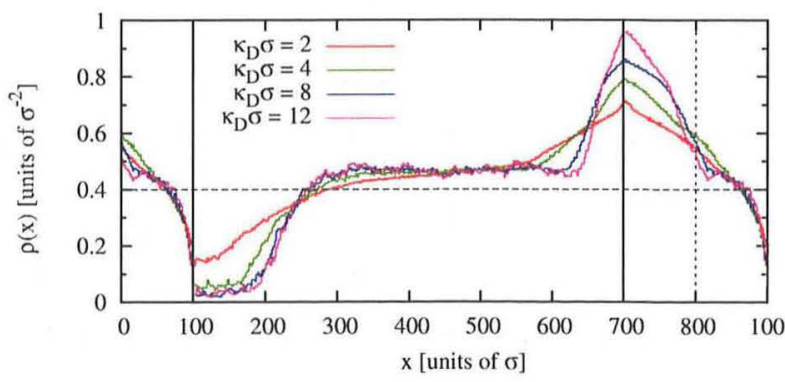

FIG. 28. (Color online) Simulation: density profiles along the channel for a selection of Debye screening lengths $\kappa_{D}$ of a YHC system $\left(\beta V_{0}=50\right)$. The driving force is applied only within the channel region $x \in[100,700] \sigma$ and the system is periodic in $x$ direction. These profiles correspond to the superimposed configurations of Fig. 27. For better clarity, we replicate the interval $x$ $\in[0,100] \sigma$ again on the right hand side of the diagram. 


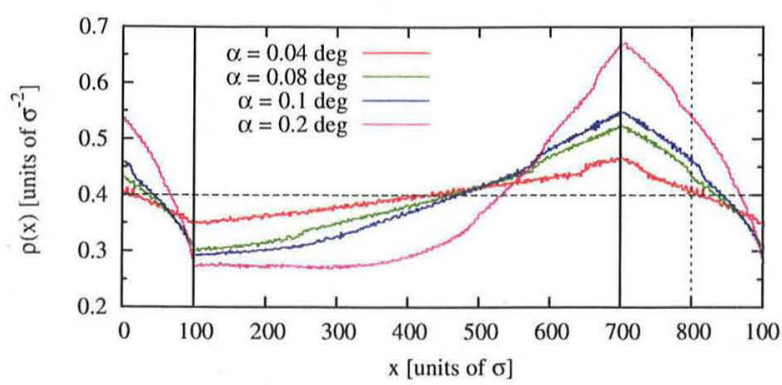

FIG. 29. (Color online) Simulation: density profiles for a selection of inclinations $\alpha$ of a system with the inclination (i.e., the driving force) applied only within the region $x \in[100,700] \sigma$. The system is periodic in $x$ direction. Shown are histograms obtained by evaluation of 1000 configurations of the system having reached a stationary nonequilibrium situation ( $\mathrm{after} \approx 2 \times 10^{6} \mathrm{BD}$ time steps). The applied magnetic field strength is $B=0.25 \mathrm{mT}, \Gamma=133.4$, and the overall particle density $n=0.4 \sigma^{-2}$. For better clarity, we replicate the interval $x \in[0,100] \sigma$ again on the right hand side of the diagram.

greater than the particle drift within the reservoir being the reason for the sharp density gradients, which lead to the sudden onset of a layered structure with eight layers in the Figs. 27 (c) and 27(d). For $\kappa_{D}=12 \sigma^{-1}$ even a layer transition to nine layers takes place due to local density values greater than $0.9 \sigma^{-2}$ which is not observed for the other three cases.

\section{ALTERNATIVE BOUNDARY CONDITIONS IN FLOW DIRECTION}

The connection of the channel to the two reservoirs has great influence on the characteristics of the stationary nonequilibrium density profile along the channel. Therefore, we performed simulations with an alternative boundary condition, where the constant external driving force only acts within the interval $x \in[100,700] \sigma$ and a periodic boundary condition is applied in $x$ direction. Figure 29 shows the resulting stationary nonequilibrium density profiles after 3 $\times 10^{6} \mathrm{BD}$ time steps for a selection of inclinations $\alpha$ of a dipolar system. For each inclination two curves are plotted which correspond to the two channel widths $L_{y}=8 \sigma$ and $L_{y}$ $=10 \sigma$. Obviously, the steady-state density profile along the channel does not depend on the channel width.

Comparison of these density profiles with those of Fig. 11 highlights the strong influence of the different realization of the reservoirs. All simulations are started from a homogeneous particle distribution of local density $\rho=0.4 \sigma^{-2}$. Instead of a density decrease we find in Fig. 29 a buildup of the local density occurring due to the filling of the reservoir at the channel end. This corresponds to the experimental situation, where the reservoir at the channel end is filled. For the small inclination $\alpha=0.04$ deg a linearly increasing density profile is obtained within the channel region. Higher inclinations lead to deviation from such a linear profile. For $\alpha=0.2 \mathrm{deg}$ a constant profile with local density $\rho \approx 0.275 \sigma^{-2}$ in $x$ $\in[100,400] \sigma$ is followed by a sharp increase in the local density up to $\rho=0.67 \sigma^{-2}$ at the channel end at $x=700 \sigma$.
In the stationary nonequilibrium state the density profile in the reservoirs can be approximated by a linear gradient. The net flux $J$ in the reservoirs fulfills Fick's law

$$
J=\frac{k_{B} T}{2 l_{0}}\left(\rho_{1}-\rho_{0}\right),
$$

where $\rho_{0} \equiv \rho(x=100 \sigma)$ and $\rho_{1} \equiv \rho(x=700 \sigma)$ are the local number densities at the channel beginning and end respectively. Due to the periodic boundary condition in $x$ direction this is equal to the net flux in the channel region $x$ $\in[100,700) \sigma$. Therefore, $J$ may be approximated by the slope of the linear density profiles in the two reservoir regions.

Figure 30 shows the layer order parameters $\Psi_{\text {layer }, n_{l}}$ for a selection of inclinations $\alpha$ in combination with the corresponding superimposed configurations. Clearly, the layer configuration and the number of layer transitions can be tuned by the strength of the driving force for the realization of the boundary condition 2 of the flow. Increasing $\alpha$ leads to multiple transitions. Interestingly, the layer transitions from seven to eight layers occur at identical $x$ positions in the Figs. 30(b)-30(d). As before, the particle flow across the position of the layer transition, which remains fixed in position.

\section{CONCLUSION}

We have reported on a variety of ordering and transport phenomena which are induced by the confinement of colloidal particles to microchannels and by the application of a constant driving force along the channel. We have analyzed the particle behavior both under equilibrium and under (stationary) nonequilibrium conditions both in experiment and by Brownian dynamics simulations.

First, we have studied the self-assembly of repulsive particles under equilibrium conditions, i.e., without a driving force applied. We have observed a boundary induced formation of a global layered structure in the channels. Such a behavior is known for a variety of related systems $[14,30,33,41,43]$. Systematically, we have analyzed the influence of the channel width $L_{y}$ and the influence of the strength of the dipolar particle repulsion. Based on the order parameter we have calculated the effect of channel width and interaction strength on laterally confined superparamagnetic particles within the solid state. The system structure varies between solidlike and liquidlike behavior when the channel width is changed. When the channel width is increased, a periodic destabilization of the layered structure with $n_{l}$ layers takes place, and the system switches to a structure with $n_{l}$ +1 layers. The bulk defect concentration $C_{\text {defect }}^{b}$ shows periodic oscillations as a function of the channel width $L_{y}$, but not as a function of the dimensionless interaction parameter $\Gamma$. The period of the oscillations is $\sim R$, where $R$ denotes the average distance of two neighboring particle layers in an unbounded hexagonal system. Such a behavior previously was reported as a result of both experiments and simulations of a similar system by Haghgooie et al. [30,31,40]. Our data show excellent qualitative and quantitative agreement with their results. 

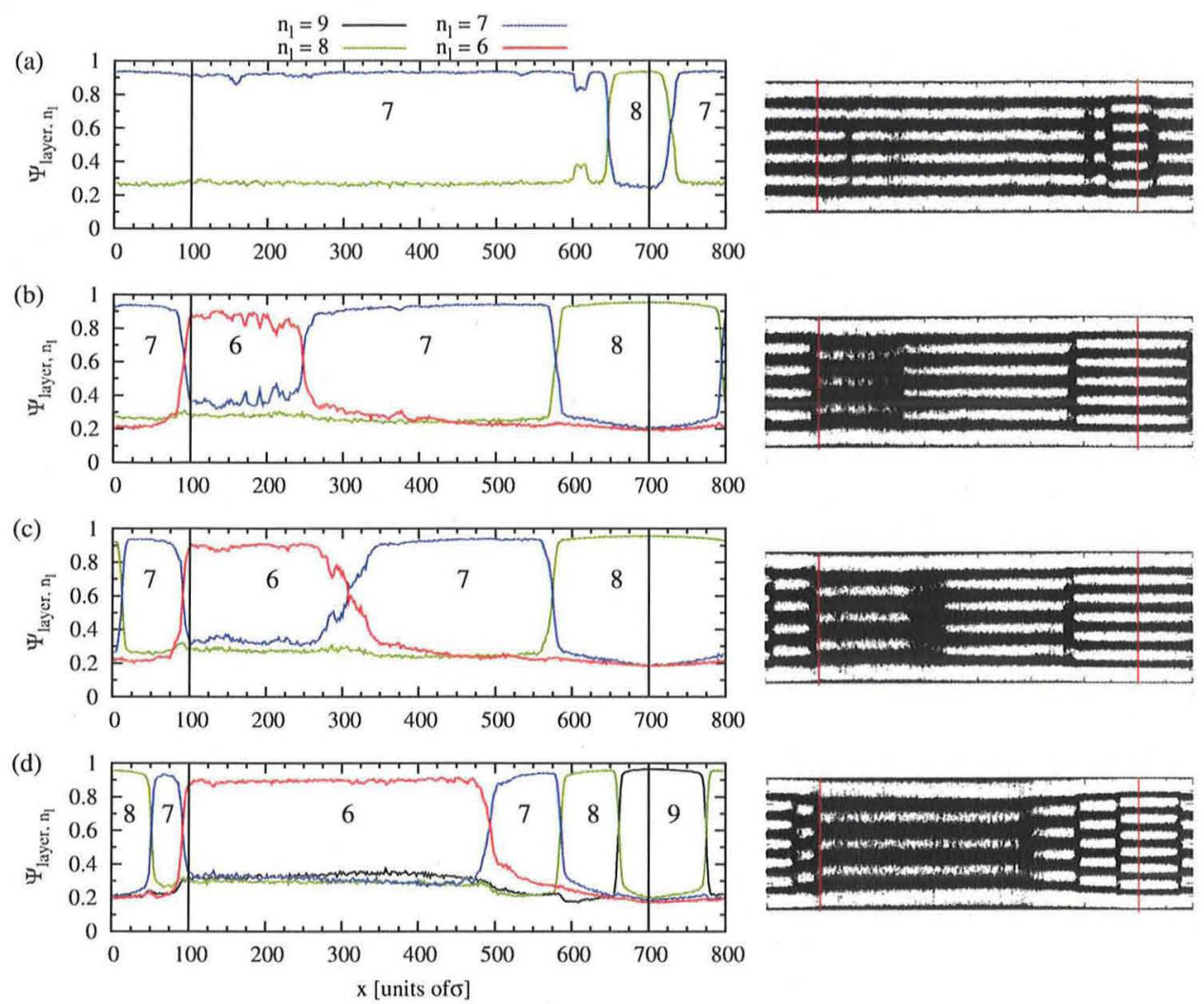

FIG. 30. (Color online) Simulation: Stationary nonequilibrium situations of systems where the driving force is applied only in $x$ $\in[100,700] \sigma$ for a selection of inclinations: (a) $\alpha=0.04 \mathrm{deg}$, (b) $\alpha=0.08 \mathrm{deg}$, (c) $\alpha=0.1 \mathrm{deg}$, (d) $\alpha=0.2 \mathrm{deg}$. For every inclination we show the average local layer order parameters $\Psi_{\text {layer, }, n_{l}}$ and the corresponding superposition of 1000 snapshots. The other simulation parameters are: $L_{x}=800 \sigma, L_{y}=10 \sigma, n=0.4 \sigma^{-2}, B=0.25 \mathrm{mT}$, and $\Gamma=133.4$. The numbers in the figures indicate the number of layers being present within a certain region.

We have shown that the number of layers can be reduced in equilibrium situations with nonparallel walls and in nonequilibrium situations with parallel walls and different boundary conditions at the channel ends. One of our main findings is that in the nonequilibrium situation the particles flow across the layer reduction zones, which remain at stable positions.

In particular, we have predicted and systematically analyzed the phenomenon of particle layer reduction under the influence of a constant driving force acting along the channel. For small driving forces $F^{\mathrm{ext}}$, where the particles are not yet in the regime of plug flow the superparamagnetic particles dynamically rearrange into different numbers of layers during transport through the channels. We have found, that along the channel the number of layers decreases gradually by steps of one. The occurrence of the layer reduction has been confirmed by the experiments. In the experiments, the massive particles sediment to the bottom of the channel due to gravity, and there they form a quasi-2D system. After having equilibrated the system, the whole setup is tilted, so that the colloidal particles are driven through a lithographically fabricated microchannel under the influence of gravity.

In very good qualitative agreement with the experiments we have shown that the reduction in layers originates from a density gradient along the channel. Quantitative differences

are expected, because the Stokes diffusion coefficient $D_{0}$, which is valid for unbounded systems and is used in the simulations, differs from the real diffusion coefficient in presence of the confinement of the experimental setup [47].

The reduction in layers takes place for specific values of the local density $\rho(x)$ and within a distance of only a few particle diameters. We have explicitly shown that the particles flow across the regions of layer reduction and thereby dynamically adjust to the local density $\rho(x)$. But additional simulation studies of systems with screened Coulomb particle interaction, where the interaction range has been varied, have shown that a longitudinal density gradient and consequently layer transitions occur for particle interaction ranges which are greater than the average distance of the particles from their neighbors. For particle pair potentials with smaller interaction ranges than the average nearest neighbor separation we observe that the layer transition region smears out, because more particle defects occur due to a smaller density gradient. No layer transitions will be observed for the model case of hard-core particles. For our choice of boundary conditions we have found, that the density gradient becomes more pronounced with decreasing inclination $\alpha$, i.e., with decreasing driving force. The density decrease is maximum at $\alpha=0.0 \mathrm{deg}$, because the particle reinsertion scheme, which we used, induces a pressure difference between both 
channel ends, even in the case when no external driving force has been applied.

Generally, we have seen both in simulations and in experiments that the local density decreases monotonically and continuously along the channel. In front of a layer transition the local structure is stretched in longitudinal direction, whereas after the layer transition the structure is longitudinally compressed and one layer has disappeared. Therefore, the local lattice constant $d_{x}(x)$ in longitudinal direction increases up to the position of the layer transition, at which it shows a noncontinuous decrease. Simultaneously, the local lattice constant $d_{y}(x)$ in transversal direction is constant in front of the layer transition, at which it jumps to the next level according to the number of layers and remains constant again. Both effects compensate each other and thus explain the continuous behavior of the local density along the channel.

By a static stretching analysis we have confirmed that a certain layered structure becomes energetically unstable and thus changes to a structure, where it has one layer less. The estimated values of the local density, where the transition takes place, are in quite good agreement with the observation. In stationary nonequilibrium the position of the layer transition oscillates about a fixed position. The amplitude of the oscillations depends on the strength of the particle interaction. We have shown, that the oscillations of the layer transition can either be analyzed by the appropriate local layer order parameters $\Psi_{\text {layer, } n_{l}}(x)$ or by the local lattice constant $d_{y}(x)$.
Each layer transition is connected to a defect, which is defined by a pair of particles with five and seven nearest neighbors, respectively. Additional periodic defects have been observed along the channel walls. Due to the purely repulsive particle interaction the edge particles are pushed against the flat walls. This leads to very small transverse fluctuations of the edge particles and a slightly higher line density of the edge particles than of the particles belonging to the layers in the central region of the channel.

It has been shown, that channel walls made of periodically fixed particles give rise to shear effects between the particles of the central layers, which move faster, than the particles, which are in the layer next to the edge particles. The latter particles show small oscillations about the average drift velocity.

The results shown concern a rather simple classical model system. The observed phenomena, however, will take place in any systems of long range interacting particles which are driven through a constriction. Therefore the results which have been gained from the studies of this system can be seen as a first step in the understanding of transport processes in many biological and quantum systems.

\section{ACKNOWLEDGMENTS}

We gratefully acknowledge the support of the SFB 513, the SFB TR6, and the NIC, HLRS, and SSC.
[1] D. Helbing, P. Molnár, I. Farkas, and K. Bolay, Environ. Plann. B Plann. Des. 28, 361 (2001).

[2] M. Rex, H. Löwen, and C. N. Likos, Phys. Rev. E 72, 021404 (2005).

[3] M. Rex and H. Löwen, Phys. Rev. E 75, 051402 (2007).

[4] M. Rex and H. Löwen, Eur. Phys. J. E 26, 143 (2008).

[5] J. Chakrabarti, J. Dzubiella, and H. Löwen, Europhys. Lett. 61, 415 (2003).

[6] J. Chakrabarti, J. Dzubiella, and H. Löwen, Phys. Rev. E 70, 012401 (2004).

[7] J. Dzubiella and H. Löwen, J. Phys.: Condens. Matter 14, 9383 (2002).

[8] M. E. Leunissen, C. G. Christova, A.-P. Hynninen, C. P. Royall, A. I. Campbell, A. Imhof, M. Dijkstra, R. v. Roij, and A. v. Blaaderen, Nature (London) 437, 235 (2005).

[9] B. J. van Wees, H. van Houten, C. W. J. Beenakker, J. G. Williamson, L. P. Kouwenhoven, D. van der Marel, and C. T. Foxon, Phys. Rev. Lett. 60, 848 (1988).

[10] D. A. Wharam, T. J. Thornton, R. Newbury, M. Pepper, H. Ahmed, J. E. F. Frost, D. G. Hasko, D. C. Peacock, D. A. Ritchie, and G. A. C. Jones, J. Phys. C 21, L209 (1988).

[11] E. Scheer, N. Agraït, J. C. Cuevas, A. Levy Yeyati, B. Ludoph, A. Martín-Rodero, G. Rubio Bollinger, J. M. van Ruitenbeek, and C. Urbina, Nature (London) 394, 154 (1998).

[12] M. Dreher, F. Pauly, J. Heurich, J. C. Cuevas, E. Scheer, and P. Nielaba, Phys. Rev. B 72, 075435 (2005).

[13] F. Pauly, M. Dreher, J. K. Viljas, M. Hafner, J. C. Cuevas, and
P. Nielaba, Phys. Rev. B 74, 235106 (2006).

[14] P. Glasson, V. Dotsenko, P. Fozooni, M. J. Lea, W. Bailey, G. Papageorgiou, S. E. Andresen, and A. Kristensen, Phys. Rev. Lett. 87, 176802 (2001).

[15] G. Piacente and F. M. Peeters, Phys. Rev. B 72, 205208 (2005).

[16] R. Roth and D. Gillespie, Phys. Rev. Lett. 95, 247801 (2005).

[17] T. M. Squires and S. R. Quake, Rev. Mod. Phys. 77, 977 (2005).

[18] K. Zahn, R. Lenke, and G. Maret, Phys. Rev. Lett. 82, 2721 (1999).

[19] K. Zahn, A. Wille, G. Maret, S. Sengupta, and P. Nielaba, Phys. Rev. Lett. 90, 155506 (2003).

[20] P. Keim, G. Maret, U. Herz, and H. H. von Grünberg, Phys. Rev. Lett. 92, 215504 (2004).

[21] C. Eisenmann, U. Gasser, P. Keim, and G. Maret, Phys. Rev. Lett. 93, 105702 (2004).

[22] R. Bubeck, C. Bechinger, S. Neser, and P. Leiderer, Phys. Rev. Lett. 82, 3364 (1999).

[23] R. Bubeck, Ph.D. thesis, University of Konstanz, Germany, 2002.

[24] P. Henseler, Master's thesis, University of Konstanz, Germany, 2002.

[25] I. V. Schweigert, V. A. Schweigert, and F. M. Peeters, Phys. Rev. Lett. 84, 4381 (2000).

[26] Q.-H. Wei, C. Bechinger, D. Rudhardt, and P. Leiderer, Phys. Rev. Lett. 81, 2606 (1998). 
[27] C. Bechinger, M. Brunner, and P. Leiderer, Phys. Rev. Lett. 86, 930 (2001).

[28] W. Strepp, Ph. D. thesis, University of Konstanz, Germany, 2001.

[29] W. Strepp, S. Sengupta, and P. Nielaba, Phys. Rev. E 66, 056109 (2002)

[30] R. Haghgooie and P. S. Doyle, Phys. Rev. E 70, 061408 (2004).

[31] R. Haghgooie, C. Li, and P. S. Doyle, Langmuir 22, 3601 (2006).

[32] A. Ricci, Ph. D. thesis, Johannes Gutenberg University, Mainz, Germany (2006).

[33] A. Ricci, P. Nielaba, S. Sengupta, and K. Binder, Phys. Rev. E 74, 010404(R) (2006).

[34] A. Ricci, P. Nielaba, S. Sengupta, and K. Binder, Phys. Rev. E 75, 011405 (2007)

[35] Y. Xia and G. M. Whitesides, Angew. Chem. Int. Ed. 37, 550 (1998).

[36] M. Köppl, P. Henseler, A. Erbe, P. Nielaba, and P. Leiderer, Phys. Rev. Lett. 97, 208302 (2006).

[37] D. L. Ermak, J. Chem. Phys. 62, 4189 (1975).

[38] M. P. Allen and D. J. Tildesley, Computer Simulation of Liquids (Oxford Science Publications, New York, 1987).
[39] D. M. Heyes and J. R. Melrose, J. Non-Newtonian Fluid Mech. 46, 1 (1993).

[40] R. Haghgooie and P. S. Doyle, Phys. Rev. E 72, 011405 (2005).

[41] L.-W. Teng, P.-S. Tu, and L. I, Phys. Rev. Lett. 90, 245004 (2003).

[42] M. Kong, B. Partoens, A. Matulis, and F. M. Peeters, Phys. Rev. E 69, 036412 (2004).

[43] G. Piacente, I. V. Schweigert, J. J. Betouras, and F. M. Peeters, Phys. Rev. B 69, 045324 (2004).

[44] P. Henseler, Ph. D. thesis, University of Konstanz, Germany, 2008.

[45] N. W. Ashcroft and N. D. Mermin, Solid State Physics (Saunders College Publishing, Philadelphia, 1976).

[46] A perfect triangular lattice fulfills the condition $d_{x} / d_{y}=2 / \sqrt{3}$. In our case an overstretched lattice is given for $d_{x} / d_{y}>2 / \sqrt{3}$. For a compressed lattice structure holds the condition $d_{x} / d_{y}$ $<2 / \sqrt{3}$.

[47] Haghgooie et al. [31] measured the surface diffusion coefficient of the colloids to be $\sim 52 \%$ of the calculated Stokes diffusion coefficient $D_{0}$ for their system, which is similar to our system. 